\title{
Estimation of Longitudinal Speed Robust to Road Conditions for Ground Vehicles
}

\author{
Ehsan Hashemi, Alireza Kasaiezadeh, Saeid Khosravani, Amir Khajepour, Nikolai Moshchuk, and Shih-Ken Chen
}

\begin{abstract}
This article seeks to develop a longitudinal vehicle velocity estimator robust to road conditions by employing a tire model at each corner. Combining the lumped LuGre tire model and the vehicle kinematics, the tires internal deflection state is used to gain an accurate estimation. Conventional kinematicbased velocity estimators use acceleration measurements, without correction with the tire forces. However, this results in inaccurate velocity estimation because of sensor uncertainties which should be handled with another measurement such as tire forces that depend on unknown road friction. The new Kalman-based observer in this paper addresses this issue by considering tire nonlinearities with a minimum number of required tire parameters and the road condition as uncertainty. Longitudinal forces obtained by the unscented Kalman filter on the wheel dynamics is employed as an observation for the Kalman-based velocity estimator at each corner. The stability of the proposed time-varying estimator is investigated and its performance is examined experimentally in several tests and on different road surface frictions. Road experiments and simulation results show the accuracy and robustness of the proposed approach in estimating longitudinal speed for ground vehicles.
\end{abstract}

Keywords-Vehicle state estimation, uncertain dynamics, stability analysis, parameter estimation, unscented Kalman filter

\section{INTRODUCTION}

Two major vehicle velocity estimation approaches are used in recent literature. The first one uses vehicle's planar kinematics, to develop observers [1], [2] that use standard measurements from an inertial measurement unit (IMU) such as acceleration, the yaw, the pitch, and the roll rates, without implementing a tire model. This approach is independent from tire parameters, but instead the sensors bias and noise should be identified precisely to have a reliable estimation. Employing accelerations and wheel speed measurements, Imsland et al. proposed a nonlinear observer in [3], [4] to tackle model uncertainties. A Kalman observer is employed by Hsu and Bevly et al. in [5], utilizing the same kinematic-based method and correction with GPS data, but the poor accuracy of the mostly practiced conventional GPSs and the loss of reception are primary concerns. The issue of reliance on a GPS is addressed with observer development around tire capacities and using the wheel speed, as investigated by G. Baffet and A. Charara [6], but this approach is incapable of predicting the lateral limit before it occurs. This drawback is eliminated in [7] by implementing a steer-by-wire system to determine the road

Ehsan Hashemi, Saeid Khosravani, and Amir Khajepour are with the Department of Mechanical and Mechatronics Engineering, University of Waterloo, ON, Canada. email: ehashemi, a.khajepour@uwaterloo.ca

Alireza Kasaiezadeh, Shih-Ken Chen, and Nikolai Moshchuki are with the R\&D Department, General Motors Co., Warren, MI, USA condition, tire limits, and the slip angles of the front and rear tracks independently. Yoon and Peng [8] utilizes two low-cost GPS receivers for the lateral state estimation and compensates the low update rate issue of the conventional GPS receivers by combining the IMU and GPS data with an extended Kalman filter $(\mathrm{EKF})$.

Another velocity estimation method uses a tire model together with vehicle kinematics. However, this approach needs road condition information and tire parameters, which may vary significantly. The issue of time-varying tire parameters is handled with a model scheduling in [9], which again lacks information on road conditions. An extended Kalman filter (EKF) is employed for both longitudinal and lateral state estimation in [10], but the load of computations justifies using a reliable approach such as an unscented Kalman filter (UKF) without any need for linearization in the system dynamics. The UKF [11], [12] and the simplified empirical Magic formula [13], [14] (as the tire model) is employed in [15] for the velocity and force estimation at each corner, but road friction and wheel torques are required for this approach. Alternatively, a sliding-mode observer is proposed in [16], based on the LuGre dynamic model [17], to estimate the longitudinal speed, but the road should be identified simultaneously. Zhang et al. presented a sliding-mode observer for velocity estimation in [18], with the wheel torques and an EKF for estimation of the Burckhardt's model [19] parameters. However, this method needs an accurate tire model, which is not possible due to the presence of tire wear, inflation pressure, and road friction uncertainties. Vehicle state estimation on a single track car model is studied for slip angle estimation in [20], with a Pacejka tire model [14]. The derivatives of the lateral forces in their approach, however, amplify noise effects in the lateral/longitudinal state estimates.

In this paper, kinematic equations are combined with the average lumped LuGre model [21] to estimate the longitudinal velocity and the tire internal deflection state at each corner independently, using a Kalman Filter (KF). Advantages of the developed velocity estimator are its robustness to road conditions and insensitivity to reasonable changes in tire parameters. The stability of the time-varying estimator and boundedness of the error covariances are also addressed in this article. Furthermore, the stability of the estimator is studied for deterministic and stochastic initial state vectors.

This paper provides different observers for the vehicle state estimation on various road conditions with the following contributions: 1) longitudinal and vertical tire-free force estimators are developed using computationally efficient nonlinear observers and common measurements without knowing the road condition and any limiting assumption. 2) a novel observer 
for the velocity estimation, robust to road friction changes is introduced which treats acceleration measurement noises and the road condition as uncertainties. 3) the corner-based structure of the longitudinal/lateral force and velocity estimators advantageously leads to better performance of the stability and traction control systems in conditions with different road friction under each tire.

This article is divided into five sections. The longitudinal force estimation approach is examined in Section II, as are corner-based estimation by UKF and unknown input observer methodologies. Section III proposes a velocity estimator with a high-slip detection module utilizing the KF. Proof of the error covariance boundedness and the stability of the linear timevarying estimator are also provided in Section III. Road test experiments and simulation results are presented in Section IV, with discussion on the findings in various maneuvers with high and low longitudinal excitations. Section V includes conclusions and future work.

\section{LONGITUdinAL FORCE ESTIMATION}

Tire forces can be measured at each corner, but their cost impact, calibration and maintenance are their major drawbacks to be used for production vehicles. Provided that the longitudinal tire force calculation needs road friction, even accurate slip ratio information from the GPS will not provide forces at each corner. Hence, estimation of tire forces independent of road conditions would be a remedy. Longitudinal force estimation independent of the road friction may be classified on the basis of wheel dynamics into the nonlinear and sliding mode observers [22]-[24], Kalman-based estimation [25], [26], and unknown input observers [27], [28]. This section provides two force estimation approaches, an unknown input observer and a Kalman-based method. The latter is selected in this study for the velocity estimation as it is less sensitive to the tire's effective radius and provides more smooth outcomes for the transient regions during acceleration and brake. Experimental and simulation results are presented in Section IV to show the estimators performance in different maneuvers and on various road friction conditions.

\section{A. Force estimation with unknown input observer}

A corner-based unknown input observer (UIO) method was proposed by Wang et al. in [28], but the stability of the error dynamics is explored in this paper. This method considers longitudinal tire forces $F_{x}$ as unknown inputs for the wheel dynamics:

$$
\dot{\omega}=\frac{1}{I_{w}}\left(T_{t}-R_{e} F_{x}-C_{b} \omega\right),
$$

where $R_{e}$ is the wheel effective radius, $T_{t}$ represents the total effective torque on the wheel at each corner, $\dot{\omega}$ is the wheel's angular acceleration, and $C_{b}$ shows the wheel bearing's linear viscous damping. The bearing viscous damping forces are negligible compared to other terms in (1). This approach provides a virtual wheel speed using a so-called Proportional, Integral, Derivative (PID)-type estimator $\dot{\hat{\omega}}=\frac{1}{I_{w}}\left(T_{t}-R_{e} f_{0}+K_{p} \tilde{\omega}\right)$ in which the constant $f_{0}$ is the nominal value of the longitudinal tire force and can be an arbitrary bounded value. The difference between the measured and the virtually estimated wheel speed $(\tilde{\omega}=\omega-\hat{\omega})$ is used for the force estimation with the proportional term, as in [28]:

$$
\hat{F}_{x}(s)=f_{0}-\frac{1}{R_{e}} K_{p} \tilde{\omega}(s) .
$$

Matching between the measured (1) and virtually estimated wheel speed $\hat{\omega}$ means that the control input produces the effect of unmeasured $-R_{e} \hat{F}_{x}$. Thus, the estimation error dynamics for the wheel speed yields:

$$
\dot{e}=-\mathcal{K} e-\mathcal{B},
$$

where $\mathcal{K}=\frac{K_{p}}{I_{w}}$ and $\mathcal{B}=\frac{R_{e}\left(F_{x}-f_{0}\right)}{I_{w}}$. The stability of error dynamics (3) is investigated in the following.

Theorem 1: The unknown input observer (2) for longitudinal force estimation is stable and the estimation error is bounded by $\frac{b}{2 \mathcal{K} \sqrt{\xi(1-\xi)}}$ where $0<\xi<1$ and $b$ is the bound for $\mathcal{B}$ in (3).

Proof: To study the stability of the error dynamics (3), the Lyapunov function $V=\frac{1}{2} e^{2}$ is used. The derivation of the Lyapunov function yields:

$$
\dot{V}=-2 \mathcal{K} V-e \mathcal{B} \leq-2 \mathcal{K} V+\|e\|\|\mathcal{B}\| .
$$

The term $\mathcal{B}$ is bounded because of the bounded characteristics of the real longitudinal force. Therefore, || $\mathcal{B}||<|b|$, and the time derivative of the Lyapunov function is developed again by introducing $0<\xi<1$ as:

$$
\begin{aligned}
\dot{V} & \leq-\mathcal{K}\|e\|^{2}+b\|e\| \\
& \leq-\mathcal{K}(1-\xi)\|e\|^{2}-\mathcal{K} \xi\|e\|^{2}+b\|e\| \\
& \leq-2 \mathcal{K}(1-\xi) V-\left(\sqrt{\mathcal{K} \xi}\|e\|-\frac{b}{2 \sqrt{\mathcal{K} \xi}}\right)^{2}+\frac{b^{2}}{4 \mathcal{K} \xi} \\
& \leq-2 \mathcal{K}(1-\xi) V+\frac{b^{2}}{4 \mathcal{K} \xi} .
\end{aligned}
$$

The term $2 \mathcal{K}(1-\xi)$ is replaced with $\gamma$, and $\mathcal{D}$ stands for $\frac{b^{2}}{4 \mathcal{K} \xi}$; then:

$$
\dot{V}+\gamma V \leq \mathcal{D}
$$

Multiplying both sides of the inequality by $e^{\gamma} t$ and then integrating results in:

$$
V-V(0) e^{-\gamma t} \leq \mathcal{D} \int_{0}^{t} e^{\gamma(\tau-t)} d \tau .
$$

Substituting the Lyapunov function $V=\frac{1}{2} e^{2}$ in (7) leads to:

$$
\frac{1}{2} e^{2} \leq V(0) e^{-\gamma t}+\frac{\mathcal{D}}{\gamma}\left(1-e^{-\gamma t}\right) .
$$

Consequently, the bound for the error can be described as:

$$
\|e\| \leq\|e(0)\| e^{-\frac{\gamma t}{2}}+\sqrt{2 \mathcal{D} / \gamma} \text {. }
$$


The term $\sqrt{\frac{2 \mathcal{D}}{\gamma}}$ is simplified to $\frac{b}{2 \mathcal{K} \sqrt{\xi(1-\xi)}}$, showing that any increase in $\mathcal{K}$ leads to a decrease in $\|e\|$. As a result, the UIO for the longitudinal force estimator (2) with the proportional term has a bounded estimation error and can be utilized whenever, wheel torques are available.

\section{B. Kalman-based force estimation}

Estimation problems can be addressed by UKF for the discrete-time nonlinear system of the form $x_{k+1}=$ $\mathcal{F}\left(x_{k}, u_{k}, f, n_{p k}\right), y_{k}=\mathcal{H}\left(x_{k}, f, n_{m k}\right)$. Parameters $f$ and system states $x_{k}$ can be estimated recursively from the noisy output $y_{k}$. Uncertainties in the process and measurements are incorporated into the nonlinear system definition as $n_{p k}, n_{m k}$. Proper capturing of nonlinearities contributes to the unscented transformation that defines the Sigma vectors $\mathcal{X} \in \mathbb{R}^{N \times 2 N+1}$, ( $N$ is the length of the state vector), which are supposed to propagate through the nonlinear system. With some minor changes, UKF can also be employed for parameter estimation instead of state estimation for the vehicle parameter identification [29], [30] and for the longitudinal force estimation [26]. For the force estimation with UKF, the effective torque $T_{t}$ provides input $u_{k}$; the wheel speed is assumed to be the state $x_{k}$, and the estimated longitudinal force $\hat{F}_{x}$ is denoted by the estimated parameter $\hat{f}$. The discrete-time parameter estimation problem can be expressed as:

$$
f_{k+1}=f_{k}+\varrho_{k}, z_{k}=\mathcal{G}\left(x_{k}, f_{k}\right)+\nu_{k},
$$

where $z_{k}$ corresponds to nonlinear observation on $f_{k}$ and $\varrho_{k}, \nu_{k}$ represent process and observation noises respectively. In a parameter estimation problem, the estimated mean is updated as $\hat{f}_{m k}=\hat{f}_{k-1}$ and initialized by $\hat{f}_{0}=$ $\mathbb{E}[f]$. The moving sample points i.e. sigma vector $F_{k \mid k-1}$ are generated around the estimated mean $\hat{f}_{m k}$ with the conventional unscented transformation pattern $F_{k \mid k-1}=$ $\left[\begin{array}{lll}\hat{f}_{m k} & \hat{f}_{m k}+\tau \sqrt{\bar{P}_{f_{k}}} & \hat{f}_{m k}-\tau \sqrt{\bar{P}_{f_{k}}}\end{array}\right]$, where square root factorization of the covariance matrix $\bar{P}_{f_{k}}$ is obtained by Cholesky decomposition at each time step $k$. The error covariance matrix of the estimated parameter is initialized with $P_{f_{0}}=\mathbb{E}\left[\left(f-\hat{f}_{0}\right)\left(f-\hat{f}_{0}\right)^{T}\right]$ and updated by $\bar{P}_{f_{k}}=P_{f_{k-1}}+$ $Q_{k-1}$ with incorporation of the process noise covariance $Q_{k-1}$. Furthermore, $\tau$ is a scalar and represents the spread of the sample points far from the mean values of random variables (states). It is defined in [31] as $\tau=\sqrt{N+\eta}$, where $\eta$ is the compound scaling parameter $\eta=\epsilon^{2} N-N$. Spread of the sample points around $\hat{f}_{m k}$ is denoted by $\epsilon=\sqrt{3 / N}$. Afterward, $\beta=2$ is introduced to employ the prior information on the Gaussian distribution of $x$. Sample points are supposed to be propagated within the system (wheel dynamics) as $\mathcal{Z}_{k \mid k-1}=\mathcal{G}\left(x_{k}, F_{k \mid k-1}\right)$, and the estimated function output $\hat{z}_{k}$ is achievable from $\hat{z}_{k}=\sum_{i=0}^{2 N} W_{i}^{m} \mathcal{Z}_{i, k \mid k-1}$. The weighting parameters are also defined by $W_{i}^{c}=W_{i}^{m}=\frac{1}{2}(N+\eta)$ for all sets $i \in\{1,2, \ldots, 2 N\}$. These parameters are $W_{0}^{c}=$ $\frac{\eta}{N+\eta}+1-\epsilon^{2}+\beta$ and $W_{0}^{m}=\frac{\eta}{N+\eta}$ for $i=0$. The updated covariance matrices are given in (11) using the measurement noise covariance $R_{k}$ :

$$
\begin{aligned}
& P_{z_{k} z_{k}}=\sum_{i=0}^{2 N} W_{i}^{c}\left(\mathcal{Z}_{i, k \mid k-1}-\hat{z}_{k}\right)\left(\mathcal{Z}_{i, k \mid k-1}-\hat{z}_{k}\right)^{T}+R_{k}, \\
& P_{f_{k} z_{k}}=\sum_{i=0}^{2 N} W_{i}^{c}\left(F_{i, k \mid k-1}-\hat{f}_{m k}\right)\left(\mathcal{Z}_{i, k \mid k-1}-\hat{z}_{k}\right)^{T}
\end{aligned}
$$

The Kalman gain is achievable, by implementing these covariance matrices as $K_{k}=P_{f_{k} z_{k}} P_{z_{k} z_{k}}^{-1}$. As a result, the updated parameter and error covariance matrices can be obtained as follows [31]:

$$
\begin{aligned}
P_{f k} & =\bar{P}_{f k}-K_{k} P_{z_{k} z_{k}} K_{k}^{T}, \\
\hat{f}_{k} & =\hat{f}_{m k}+K_{k}\left(z_{k}-\hat{z}_{k}\right),
\end{aligned}
$$

where $\hat{f}_{k}$ is the updated longitudinal force estimate $\hat{F}_{x_{i j}}$ at each corner. Outcomes of the UIO and UKF approaches are compared in Section IV. The UKF moving sigma points through the wheel dynamics greatly reduce the estimation fluctuations, even with the presence of major uncertainties such as the road friction and changes in the effective radius. Therefore, longitudinal force estimation with UKF is selected in this study and utilized for the velocity estimation.

Estimated longitudinal forces should be normalized to be utilized in the velocity estimator, which will be explored in the next section. In order to normalize the longitudinal forces, normal (vertical) forces at each corner (tire) should be calculated using lateral and longitudinal vehicle dynamics. One can write the vertical and longitudinal acceleration components of the longitudinal dynamics as $\bar{a}_{\theta x}=a_{x m} \cos \theta_{v}+a_{z m} \sin \theta_{v}$ and $\bar{a}_{\theta z}=a_{z m} \cos \theta_{v}-a_{x m} \sin \theta_{v}$ in which $a_{x m}, a_{z m}$ are the measured longitudinal and vertical accelerations by an IMU attached to the sprung mass. These measurements are affected by the kinematics of the vehicle's CG, vehicle pitch angle $\theta_{v}$, and the road grade angle $\theta_{r}$. The sprung mass roll and pitch are not achievable by integration over the roll and pitch rate signals $\dot{\phi}_{m}, \dot{\theta}_{m}$ because of sensor drift. Rehm provided a linear observer with low-pass filtering in [32] to estimate the vehicle body's roll/pitch angles as $\dot{\hat{\phi}}_{v}=\dot{\phi}_{m}+L_{\phi} e_{\phi}, \dot{\hat{\theta}}_{v}=\dot{\theta}_{m}+L_{\theta} e_{\theta}$ by compensation over the error between the filtered estimates and the stationary roll/pitch values i.e. $e_{\phi}, e_{\theta}$ and the observer gains $L_{\phi}, L_{\theta}$. Normal forces at front and rear axles thus can be calculated by:

$$
\begin{aligned}
& \hat{F}_{z f}=-\frac{m}{L_{w b}} h_{C G} \bar{a}_{\theta x}+\frac{m}{L_{w b}} b_{r} \bar{a}_{\theta z}, \\
& \hat{F}_{z r}=\frac{m}{L_{w b}} h_{C G} \bar{a}_{\theta x}+\frac{m}{L_{w b}} b_{f} \bar{a}_{\theta z},
\end{aligned}
$$

where the subscripts $f, r$ represent the front and rear axles, $h_{C G}$ is the height of the vehicle's center of gravity, and the vehicle's body pitch is denoted by $\theta_{v}$. The wheel base is denoted by $L_{w b}=b_{f}+b_{r}$ in which $b_{f}, b_{r}$ are the distances from the front and rear axles to CG. Similarly, the vertical and lateral acceleration components of the lateral dynamics are $\bar{a}_{\phi y}=$ $a_{y m} \cos \phi_{v}+a_{z m} \sin \phi_{v}$ and $\bar{a}_{\phi z}=a_{z m} \cos \phi_{v}-a_{y m} \sin \phi_{v}$ in which $a_{y m}$ is the measured lateral acceleration by IMU which contains the kinematics of the vehicle's CG, vehicle roll $\phi_{v}$ 
angle, and the road bank angle $\phi_{r}$. Therefore, using (13) and equivalent masses at each axle $m_{f}=\frac{F_{z f}}{g}, m_{r}=\frac{F_{z r}}{g}$, normal forces at each corner are as follows:

$$
\begin{aligned}
& \hat{F}_{z i L}=\frac{m_{i}}{T_{i}}\left[\bar{a}_{\phi z}\left(\frac{T_{i}}{2}-h_{r c} \sin \phi_{v}\right)-\bar{a}_{\phi y} h_{C G}\right], \\
& \hat{F}_{z i R}=\frac{m_{i}}{T_{i}}\left[\bar{a}_{\phi z}\left(\frac{T_{i}}{2}+h_{r c} \sin \phi_{v}\right)+\bar{a}_{\phi y} h_{C G}\right],
\end{aligned}
$$

in which $i \in\{f, r\}$ (front and rear axles), $T_{f}, T_{r}$ represent the length of front and rear tracks respectively, and $h_{r c}$ is the height of the roll center. Normalized longitudinal forces at each tire then can be written as $\mu_{x_{i j}}=\frac{\hat{F}_{x_{i j}}}{\hat{F}_{z_{i j}}}$ where $j \in\{L, R\}$ shows the left and right tires. The normalized estimated forces $\mu_{x_{i j}}$ are used for the proposed velocity estimator and will be described in the next section

\section{LONGITUDINAL VELOCITY ESTIMATION}

Kinematic-based velocity estimators and their limitations due to lack of observation are investigated first in this section. Afterwards, a velocity estimation method that includes observations on tire forces is provided. However, the performance of the velocity estimators based on tire forces is practically limited because of uncertain road friction and tire parameters, especially for the transient and saturation regions. Consequently, this section proposes a method that assumes road condition as uncertainty and estimates velocity with a timevarying Kalman filter. The stability of such linear time-varying observer with deterministic and stochastic initial conditions is also shown.

\section{A. Kinematic-based velocity estimation}

Because of difficulties in dealing with time-varying tire parameters and unknown road conditions, conventional kinematic-based velocity estimators employ acceleration measurement and rely on GPS data intermittently. Linear, Kalman, or nonlinear observers are used in such kinematic-based methods [1], [5], [6] without using a tire model. Kinematic-based estimation structure uses transformed kinematic equations at each corner with the following dynamics:

$$
\begin{aligned}
& \dot{u}_{x}=a_{x}+r u_{y}+a_{x b}+\Omega_{x}, \\
& \dot{u}_{y}=a_{y}-r u_{x}+a_{y b}+\Omega_{y},
\end{aligned}
$$

where the yaw rate is denoted by $r$, and the vehicle states $u_{x}, u_{y}$ are estimated using the measurements of acceleration and wheel speed. The bias and uncertainties in the acceleration measurements are represented by $a_{x b}, a_{y b}$ and $\Omega_{x}, \Omega_{y}$, respectively. Any stochastic estimator may be used for (15) in which the only reliable and available measurement is the wheel speed because of the lack of a tire model which could provide tire forces as an additional measurement.

The integration of acceleration measurement, removing bias and noises by GPS, and correction with the Kalman observer would consider providing vehicle states accurately [5], however, the accurate GPS data may not be available at all time and on production vehicles due to high costs. Poor accuracy and low bandwidth of available commercial GPSs, particularly in the lateral direction, and loss of reception are primary impediments. Moreover, estimation errors for low-excitation conditions with continuous high-slip ratio in such kinematicbased techniques makes the model-based estimation methods more promising [10], [15], [16]. However, model-based approaches based on the correction with the tire forces should deal with the varying model (tire) parameters and unknown road friction. This issue is tackled in the next subsection using the lumped LuGre tire model without the road friction term.

\section{B. Corner-based velocity estimation}

Implementing a tire model independent of road conditions is desirable for velocity estimation. Doing so is possible with use of an observer-based approach, which treats the road friction as a bounded uncertainty. This method is targeted in this article and experimentally tested for both dry and slippery road conditions. The average lumped LuGre model is introduced in [21] to obtain the tire forces using the force distribution coefficient $\kappa$, rather than by integrating bristle element forces over the tire patch length. Representing average deflection of the bristles, the tire internal state $z(\zeta, t)$ in the average lumped LuGre model, has the capability of being used in a velocity estimator as a state, because of its dynamics:

$$
\dot{z}_{l}(\zeta, t)=V_{r l}-\left(\kappa_{l} R_{e}|\omega|+\frac{\sigma_{0 l}\left|V_{r l}\right|}{\theta g\left(V_{r l}\right)}\right) z_{l}(\zeta, t),
$$

in which $l \in\{x, y\}, \zeta$ is the axis coordinate, $\sigma_{0 l}$ is the rubber stiffness, $V_{r l}$ shows the relative velocities. The level of tire and road adhesion is represented by introducing a so called "Road Classification Factor", which may vary between $0<$ $\theta \leq 1$ according to dry, wet, and icy conditions respectively. The longitudinal relative velocity can be replaced by $V_{r x}=$ $R_{e} \omega-u_{x t}$ where the longitudinal velocity in the tire coordinate system is denoted by $u_{x t}$. The longitudinal relative velocity in the tire coordinate of the LuGre model resembles the slip ratio $\lambda=\frac{R_{e} \omega-u_{x t}}{\max \left\{R_{e} \omega, u_{x t}\right\}}$ in the mostly used tire models such as Burckhardt [19] and Pacejka [33]. The parameter $\kappa$ in the average lumped model can be a constant, or a function of time, or may be approximated by an asymmetric trapezoidal scheme. The suggested value for $\kappa$ in [34] is $\kappa=\frac{7}{6} L$, where $L$ is the tire patch length. In addition, $g\left(V_{r l}\right)$ is defined by $g\left(V_{r l}\right)=\mu_{c}+\left(\mu_{s}-\mu_{c}\right) e^{-\left|\frac{V_{r l}}{V_{s}}\right|^{\alpha}}$, in which $\mu_{c}, \mu_{s}$ are the normalized Coulomb friction and static friction, respectively. The Stribeck velocity $V_{s}$ shows the transition between these two friction states. The tire parameter $\alpha=0.5$ is assumed for this study.

The final form of the normalized friction force $\left(\mu_{l}=\frac{F_{l}}{F_{n}}\right)$ of the average lumped LuGre model yields $\mu_{l}=\sigma_{0 l} z_{l}+\sigma_{1 l} \dot{z}_{l}+$ $\sigma_{2 l} V_{r l}$. In addition, the internal state $z_{l}$ can be expressed using the two-dimensional combined-slip LuGre model, which considers the effect of longitudinal/lateral slip on the tire capacity in another direction. The LuGre non-measurable longitudinal internal state can be written at each corner (wheel) in the presence of uncertainty $\Omega_{z}(t)$ as:

$$
\dot{z}_{x}(\zeta, t)=V_{r x}-\kappa_{x} R_{e}|\omega| z_{x}(\zeta, t)+\Omega_{z}(t) .
$$


Uncertainty $\Omega_{z}(t)$ is replaced with the road friction term $\frac{\sigma_{0 x}\left|V_{r x}\right|}{\theta g\left(V_{r x}\right)} z_{x}$ and is bounded because of the passivity of the LuGre model. Moreover, the derivative of the relative velocity is also corrupted due to the sensor noise and bias [35]:

$$
\dot{V}_{r x}=R_{e} \dot{\omega}_{s}-\dot{u}_{x t}+\Omega_{a}(t),
$$

in which $\dot{\omega}_{s}$ stands for the wheel's rotational acceleration and $\dot{u}_{x t}$ represents the projected longitudinal acceleration in the tire coordinate system. The term $\Omega_{a}(t)$ shows the deviation of the measured relative acceleration $R_{e} \dot{\omega}_{s}-\dot{u}_{x} t$ from $\dot{V}_{r x}$ because of the sensor noises. Establishing these equations allow the development of a Kalman-based observer to incorporate longitudinal tire deflections (17) and relative velocities (18) concurrently and to compare the output with the longitudinal forces $\hat{\mu}_{x}$ provided in section II. The general form of the velocity estimator at each corner $i j$, which can be addressed by the KF, with $\omega>0$ is given as follows in which $\boldsymbol{\Omega}=\left[\begin{array}{ll}\Omega_{z} & \Omega_{a}\end{array}\right]^{T}$ :

$$
\begin{aligned}
\dot{x} & =A(t) x+B(t) u+\Omega \\
& =M^{-1}\left[\begin{array}{ccc}
-\kappa_{x} R_{e} \omega & 1 & 0 \\
0 & 0 & 0 \\
-\kappa_{x} R_{e} \dot{\omega} & 0 & -\kappa_{x} R_{e} \omega
\end{array}\right]\left[\begin{array}{c}
z_{x} \\
V_{r x} \\
\dot{z}_{x}
\end{array}\right]+M^{-1}\left[\begin{array}{l}
0 \\
1 \\
0
\end{array}\right] \\
y & =C x+\Gamma=\sigma_{0 x} z_{x}+\sigma_{2 x} V_{r x}+\sigma_{1 x} \dot{z}_{x}+\Gamma .
\end{aligned}
$$

Process and measurement noises are denoted by $\Omega, \Gamma$ respectively, and $M=\left[\begin{array}{lllllll}1 & 0 & 0 ; 0 & 1 & 0 ; 0 & -1 & 1\end{array}\right]$. The linear time-varying system (19) uses a reduced number of tire parameters: normal force distribution factor $\kappa$, rubber stiffness $\sigma_{0 x}$, rubber damping $\sigma_{1 x}$, and relative viscous damping $\sigma_{2 x}$. These tire parameters are not related to the road condition and friction parameters. The bounded error covariance and stability of linear time-varying estimators for both known zero and nonzero initial error covariance were explored in [36], [37].

Observability is a sufficient condition for implementation of an optimal variance filter (such as a Kalman estimator). On the other hand, the controllability property guarantees asymptotic stability of such filters for the linear time-invariant case. The observability and controllability conditions are next studied for the Linear Time-Varying (LTV) system (19), which has the discrete-time form:

$$
\begin{aligned}
x_{k+1} & =A_{k} x_{k}+B_{k} u_{k}+\varrho_{k}, \\
y_{k} & =C_{k} x_{k}+\nu_{k},
\end{aligned}
$$

with process and measurement uncertainties $\varrho_{k}, \nu_{k}$, which have the covariance $\bar{Q}_{k}=\mathbb{E}\left[\varrho_{k}, \varrho_{k}^{T}\right], \bar{R}_{k}=\mathbb{E}\left[\nu_{k}, \nu_{k}^{T}\right]$ accordingly. Process and measurement noises are assumed to be uncorrelated $\mathbb{E}\left[\varrho_{k}, \nu_{k}^{T}\right]=0$ and have zero mean $\mathbb{E}\left[\varrho_{k}\right]=$ $\mathbb{E}\left[\nu_{k}\right]=0 ; \forall k \in \mathbb{N}$.

Discretization of the system is done by the Step-Invariance method, because of its precision and response characteristics, which prohibit divergence of the integration of stable dynamic systems [38]. Input to the continuous-time system is the hold signal $u_{k}=u\left(t_{k}\right)$ for a period between $t_{k} \leq t<t_{k+1}$ with the sample time $T_{s}$. Then, the discrete-time system is defined by $x_{k+1}=A_{k} x_{k}+B_{k} u_{k}$ with state transition and input matrices $A_{k}=e^{A(t) T_{s}}$ and $B_{k}=\int_{0}^{T_{s}} e^{A(t) \tau} B(t) d \tau$.
The discrete-time Kalman observer suggests the following prediction with correction to estimate the states (tire internal state $z$ and the longitudinal relative velocity $V_{r x}$ ) defined by $\hat{x}_{k+1 \mid j} \triangleq \mathbb{E}\left[x_{k} \mid y_{j}\right]$ using a sequence of measurements $y_{j}$ :

$$
\hat{x}_{k+1 \mid k}=A_{k} \hat{x}_{k \mid k-1}+B_{k} u_{k}+K_{k}\left(y_{k}-C_{k} \hat{x}_{k \mid k-1}\right),
$$

where the optimal Kalman gain is $K_{k}=$ $A_{k} \bar{P}_{k \mid k-1} C_{k}^{T}\left(C_{k} \bar{P}_{k \mid k-1} C_{k}^{T}+\bar{R}_{k}\right)^{-1}$ and error covariance $\bar{P}_{k+1 \mid k} \triangleq \operatorname{cov}\left(x_{k+1}-\hat{x}_{k+1 \mid k}\right)$ forms a discrete time-varying Riccati equation (22) for both zero and non-zero state initialization $\hat{x}_{0 \mid-1}=\mathbb{E}\left[x_{0}\right]$ and covariance initialization $\bar{P}_{0 \mid-1} \triangleq \operatorname{cov}\left(x_{0}\right)=\mathbb{E}\left[\left(x_{0}-\hat{x}_{0 \mid-1}\right)\left(x_{0}-\hat{x}_{0 \mid-1}\right)^{T}\right]:$

$$
\bar{P}_{k+1 \mid k}=A_{k} \bar{P}_{k \mid k-1} A_{k}^{T}+\bar{Q}_{k}-K_{k} C_{k} \bar{P}_{k \mid k-1} A_{k}^{T} .
$$

The Kalman gain and error covariance do not depend on the measurements, even for the time-varying case, but only on the noise statistics. The estimation error is defined by $e_{k+1 \mid j} \triangleq$ $x_{k+1}-\hat{x}_{k+1 \mid j}$, which yields:

$$
e_{k+1 \mid k}=\left(A_{k}-K_{k} C_{k}\right) e_{k \mid k-1}-K_{k} \nu_{k}+\varrho_{k} .
$$

$\omega_{s}$ Stability $\rho\{$, the estimator is investigated in the following.

\section{Stability of the estimator}

Stability of the error dynamics (23) is studied in this section with known and uncertain initial conditions. The detectability and stabilizability definitions in (A1) and (A2) are required for the stability analysis of the suggested discrete-time estimators. Uniform detectability leads to bounded error covariance. In addition, stabilizability of the paired state transition matrix and process noise results in exponential stability of the estimator, as proved in [39], [40].

The stochastic observability, stability and convergence of the state mean, and bounds on error covariance of the Kalman estimator for LTV systems, such as that in (21), were studied in [39], [41]. These studies were focused on systems with deterministic parameters and known initial state vectors and done in terms of uniform complete observability and controllability grammians. On the other hand, the bounded error covariance and stability of the Kalman estimator for systems with completely uncertain initial covariance/states is investigated in [42].

Uniform detectability and stabilizability conditions are investigated in this section to check the stability and error covariance boundedness of the proposed velocity estimator for two cases: a) known zero/nonzero initial states b) complete uncertainty of the initial-state statistics.

Proposition 1: There exists a state estimator such as Kalman (21) having bounded error covariance for time-variant system (19) with deterministic time-varying parameters and known initial state/covariance.

Proof: For the system $x_{k+1}=A_{k} x_{k}+\varrho_{k}, y_{k}=$ $C_{k} x_{k}+\nu_{k}$ with uniform detectability of $\left[A_{k}, C_{k}\right]$, the known initial state/covariance, and the process and measurement noise covariances $\bar{Q}_{k}, \bar{R}_{k}$, there exists a state estimator such as the Kalman having bounded error covariance (see Appendix) [39]. Furthermore, stabilizability of the pair $\left[A_{k}, G_{k}\right]$ leads to 
exponential stability of the $\mathrm{KF}$, where $G_{k}$ is an appropriate matrix obtained by $Q_{k}=G_{k} G_{k}^{T}$. Proof is provided in [39].

Therefore, the detectability condition (A1) should be examined for the proposed velocity estimator (19). This is experimentally checked for the deterministic time-varying wheel speed of the discrete-time systems matrix $A_{k}$. The rank of $\mathbb{V}(0, N)$ on several road experiments is shown in Fig. 1, where $N$ represents the total number of iterations with the sample time $T_{s}=0.005[s]$.

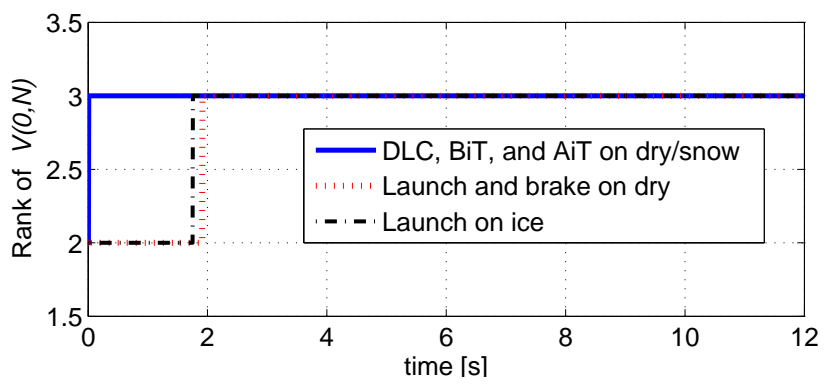

Fig. 1: Observability test: rank of $\mathbb{V}(0, N)$ for known initial state/covariance

Fig. 1 shows that the suggested estimator has full rank on the observability grammian (A1) for all performed maneuvers, showing the observability of the system for known initial covariances. Maneuvers include double lane change (DLC); acceleration-in-turn (AiT); brake-in-turn (BiT); and launch on dry, snowy, and icy roads. The launch maneuvers do not have the full rank condition for $t \leq 2$ because the wheel speed has been zero for that period, which is not the concern of this study. The stabilizability condition is also required since states should be affected by the noise such that the optimal Kalman estimator is forced to utilize measurements. This condition is also satisfied and the grammian (A2) has full rank. Additionally, since the system matrix $A(t)$ in (19) is physically bounded (because of the wheel speed and its derivative characteristics), a conventional observability test is performed here. The observability matrix for system (19) is given by [43]:

$$
\begin{array}{r}
\mathcal{O}_{n}=\left[\begin{array}{lll}
\tau_{1} & \tau_{2} \ldots & \tau_{n}
\end{array}\right]^{T}, \\
\tau_{1}=C, \quad \tau_{i+1}=\tau_{i} A(\omega)+\dot{\tau}_{i} .
\end{array}
$$

Observability is confirmed by holding the full rank condition $\operatorname{rank}\left(\mathcal{O}_{3}\right)=3$ at each fixed time span for operating regions of the wheel speed and its time derivatives. Thus, the suggested parameter-varying corner-based estimator (19) is observable and it is feasible to observe the longitudinal tire internal states $\hat{z}_{x}$ and the relative velocity $\hat{V}_{r x}$ with any known initial covariance matrix and by employing the longitudinal force as the output.

In the case of complete uncertainty on the initial state/covariance, the estimated covariance matrices can be unbounded even if the LTV system satisfies the observability criteria (A1). The bounded error covariance and stability of the Kalman filter for the proposed velocity estimator (19) with completely uncertain initial state/covariance is investigated here in the following.

Proposition 2: The longitudinal states $z_{x}, V_{r x}$ of the timevarying system (19) can be recovered using measurements $\mu_{x_{i j}}$ and a Kalman estimator with stochastic initial covariance/states at each corner.

Proof: By definition, the system (20) is stochastically observable if there exists a finite time $t_{f}$, such that the state covariance matrix $\bar{P}_{k}$ is bounded [42]:

$$
\lambda_{\max }\left(\bar{P}_{k}\right)<\lambda_{b}, t_{k} \geq t_{f}
$$

where $\lambda_{\max }\left(\bar{P}_{k}\right)$ shows the largest singular value of the matrix $\bar{P}_{k}$ and $\lambda_{b}$ is a predefined scalar bound. Assuming initial state covariance matrix $\bar{P}_{0 \mid-1}=\psi I, \psi \in \mathbb{R}, \psi>0$, one can rewrite the time-varying Riccati equation (22) as [42]:

$$
\bar{P}_{k+1 \mid k}=\psi \mathcal{M}_{k+1}+\mathcal{N}_{k+1}+\mathcal{S}_{k+1},
$$

where $\mathcal{N}_{k+1}=\mathcal{N}_{k+1}\left(\mathcal{M}_{k}, \mathcal{N}_{k}, \phi_{k}, C_{k} \bar{Q}_{k}, \bar{R}_{k}\right)$, $\mathcal{S}_{k+1}=\mathcal{S}_{k+1}\left(\psi, \mathcal{S}_{k}, \mathcal{N}_{k}, \phi_{k}, \mathcal{M}_{k}, C_{k}, \bar{Q}_{k}, \bar{R}_{k}\right)$, and $\mathcal{M}_{k+1} \triangleq \phi_{k, 0} X_{0, k} X_{0, k}^{T} \phi_{k, 0}^{T}$. The procedure for obtaining $X_{0, k}$ is provided in the Appendix and $\phi_{i, j}=\phi_{i, i-1} \phi_{i-1, j}$ are the state transition matrices for $i \geq j$ with $\phi_{i+1, i}=A_{i}$. In summary, the following Lemma presents two tests for observability of the velocity estimator with stochastic initial conditions.

Lemma 1: [42] The Kalman estimator (21) on the system (20) with an error covariance matrix (22) and stochastic initial state $\bar{P}_{0 \mid-1}=\psi I, \psi \in \mathbb{R}^{+}$is stochastically observable if the condition $\lambda_{\max }\left(\mathcal{M}_{f}\right)=0$ (test 1) holds for a finite time $t_{f}$ and $\lambda_{\max }\left(\mathcal{N}_{k+1}\right)<\lambda_{b}$ for $t_{k} \geq t_{f}$ (test 2) with a predefined bound $\lambda_{b}$, where $\mathcal{M}_{k+1}$ is obtained from the modified Riccati equation (26) and the procedure provided in (A6), (A7) in the Appendix. Employing the condition $\lambda_{\max }\left(\mathcal{M}_{f}\right)=0$ for a finite time $t_{f}<\infty$, the modified Riccati equation (26) changes to $\bar{P}_{k+1 \mid k}=\mathcal{N}_{k+1}+\mathcal{S}_{k+1}$ which leads to a simplified form of $\mathcal{N}_{k+1}$ as in:

$$
\mathcal{N}_{k+1}=A_{k} \mathcal{N}_{k} A_{k}^{T}+\bar{Q}_{k}-A_{k} \mathcal{N}_{k} C_{k}^{T} \Xi_{k}^{-1} \Xi_{k}^{-T} C_{k} \mathcal{N}_{k} A_{k}^{T},
$$

where $\Xi_{k}$ is expressed in (A7).

Whenever the two criteria on $\mathcal{M}_{f}, \mathcal{N}_{k+1}$ in Lemma 1 (so called test 1 and test 2 ) are met, the Kalman observer (21) is stable even if the scalar $\psi$ has infinite values. These two tests have been performed on the proposed observer with $\bar{Q}=2.5 e-3$ and $R=1 e-3$ and results are depicted as follows. Figure 2 exhibits $\lambda_{\max }\left(\mathcal{M}_{f}\right)$, where $\mathcal{M}_{f}$ is obtained from (26) and the procedure provided in the Appendix. Different experiments such as DLC, brake-in-turn (BiT), sharp turn, and steering on dry and slippery (snow/ice) roads have been performed and results are illustrated in Fig. 2.

From the plots in Fig. 2, it is apparent that the largest singular value of $\mathcal{M}_{f}$ converges to zero after $t_{f}=0.03 \mathrm{sec}$. for different experiments. The values of $\lambda_{\max }\left(\mathcal{N}_{k+1}\right)$ with $\mathcal{N}_{k+1}$ from (27) are plotted in Fig. 3.

The results on different road conditions and pure/combined slip maneuvers, as shown in Fig. 3, indicate that the maximum 


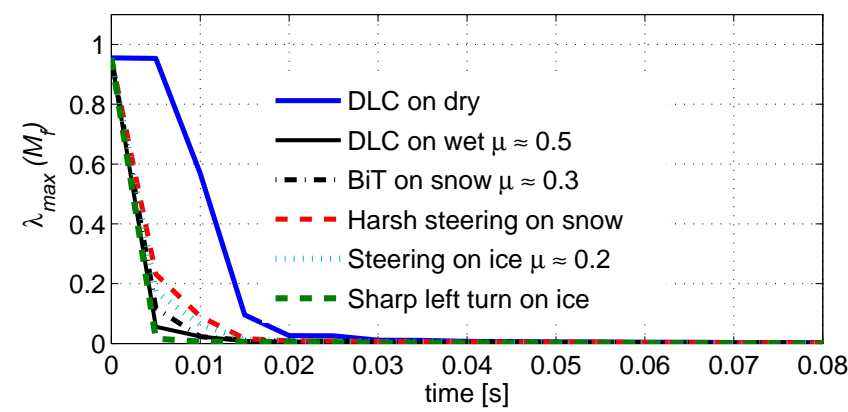

Fig. 2: Test1 for various road tests, experimental results

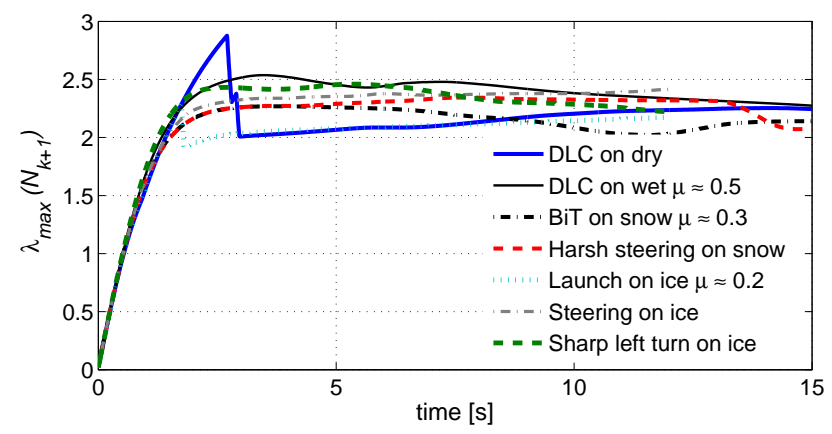

Fig. 3: Test2 for different driving scenarios and roads, experimental results

singular value of $\mathcal{N}_{k+1}$ remains bounded. Thus, the both criteria (test 1 and test 2 ) are met and the discretized form of system (19) with KF estimator (21) is stochastically observable.

Consequently, the presented model-based estimation is stable, and errors of the state mean have bounded variance for both known and stochastic initial covariance.

Figure 4 shows the structure of the discussed estimator, in which subscript $i j$ represents the estimation/measurement at each corner. The newly proposed longitudinal state estimation approach, which is independent from the road condition, is developed using the UKF force estimator (in Section II) and the Kalman observer on the linear time-varying system (19).

The presented UKF force estimator structure in Fig. 4 depicts the procedure for the sigma points propagation and the covariance matrix update. The estimated relative longitudinal velocities $\hat{V}_{r x_{i j}}$ at each corner from (21) are used for the longitudinal velocity estimation at the tire coordinates as $\hat{u}_{x t_{i j}}=R_{e} \omega_{i j}-\hat{V}_{r x_{i j}}$. Afterward, each corner's longitudinal velocity in the vehicle coordinates $\hat{u}_{x_{i j}}$ yields:

$$
\hat{u}_{x_{i j}}=\hat{u}_{x t_{i j}} \cos \delta-\hat{u}_{y t_{i j}} \sin \delta_{i j}
$$

in which $\delta_{i j}$ is the steering angle at corners and the estimated lateral velocity at each corner's tire coordinates is denoted by $\hat{u}_{y t_{i j}}$. Consequently, longitudinal velocity at the vehicle's center of gravity can be expressed as $\hat{u}_{x}=\eta_{1} \hat{u}_{x_{f}}+\eta_{2} \hat{u}_{x_{r}}$ in terms of corners' estimated velocities in which the average estimated velocities on the front axle are defined by $\hat{u}_{x_{f}}=\left(\hat{u}_{x_{f L}}+\hat{u}_{x_{f R}}\right) / 2$. Front and rear axle gains are denoted

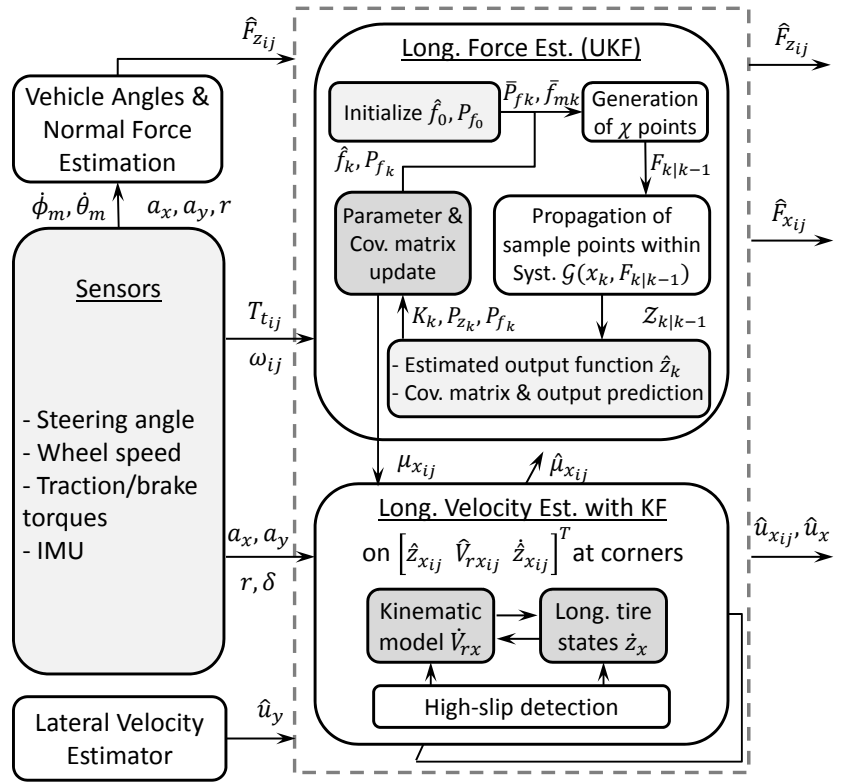

Fig. 4: Structure of the longitudinal velocity estimation with the tire model

by $\eta_{1}, \eta_{2}$. The allocated gains for the road experiments in this study are $\eta_{1}=\eta_{2}=0.5$, but they can be variable and defined adaptively based on the level of excitation.

A high-slip detection algorithm is also used to deal with the noises associated with large slip ratio conditions. Noise covariance matrices change appropriately upon detection of a high-slip case to incorporate changing in the level of reliance on the vehicle kinematics (process) and longitudinal forces (measurement). Covariance matrices $\bar{Q}, \bar{R}$ change adaptively to avoid fluctuations (caused by nonlinearities/disturbances) during harsh maneuvers on slippery surfaces.

This algorithm needs a slip ratio threshold $\bar{\lambda}_{t h}$ after which the process and measurement covariance matrices change to $\bar{Q}=3.3 e^{-4}$ and $\bar{R}=2.1 e^{-1}$ respectively. Sudden changes in the slip-ratio (vehicles response) will not be detected in case of large constant high-slip threshold. This leads to more required time for the estimated slip-ratio to satisfy the threshold (i.e. it requires larger excitations). On the other hand, small constant threshold results in unnecessary detection of the large slip cases. Thus, in the developed high-slip detection module, the threshold changes between the predefined upper and lower bounds $\lambda_{u}, \lambda_{l}$ according to the driving conditions as:

$$
\bar{\lambda}_{t h}=\lambda_{u}-\frac{1}{\varphi_{e} \sqrt{2 \pi}} e^{-\frac{\beta_{e} \sigma_{e}}{2 \varphi_{e}^{2}}}
$$

where $\varphi_{e}=\frac{1}{\sqrt{2 \pi}}\left(\lambda_{u}-\lambda_{l}\right)$ and $\sigma_{e}$ represents variance of the vehicle's acceleration $a_{k}$ over a moving window with size $N_{a}$ i.e. $\sigma_{e}=\operatorname{var}\left\{\left\|a_{k}\right\|_{2}: m-N_{a} \leq k \leq m\right\}, \forall m \in \mathbb{N}, m \geq N_{a}$, in which $\left\|a_{k}\right\|_{2}=\sqrt{a_{x_{k}}^{2}+a_{y_{k}}^{2}}$ and $a_{x_{k}}, a_{y_{k}}$ are measured longitudinal and lateral accelerations. The rate of transition between the predefined upper and lower thresholds $\lambda_{u}, \lambda_{l}$ is 
denoted by $\beta_{e}$. Thus, for $|\lambda| \geq \bar{\lambda}_{t h}$ the covariance matrices $\bar{Q}, \bar{R}$ change to the new values.

To use the current measurement and remove the outliers and detect the large slip scenarios, time derivative of the wheel speed $|\dot{\omega}|$ is also implemented and a threshold $\left|\dot{\omega}_{t h}\right|$ is introduced as a criteria after which the changes in the covariance matrices should happen. Finally, the covariance matrices change when each of the slip-based or wheel speedbased criteria are met i.e. $\left\{|\lambda| \geq \bar{\lambda}_{t h} \vee|\dot{\omega}| \geq\left|\dot{\omega}_{t h}\right|\right\}$. This leads to the prompt detection and consequently proper covariance matrix allocation. Simulation and experimental results in the next section confirm the validity of the algorithm on dry and slippery roads.

\section{RESUltS AND DiscusSiON}

This section includes simulation and experimental tests for validation of the longitudinal state estimator on a fully electrified sport utility vehicle (SUV) with four independent wheel drive, specifications given in Table I, and longitudinal tire parameters $\sigma_{0 x}=612, \sigma_{1 x}=0.87, \sigma_{2 x}=0.0016, \kappa=8$, and $R_{e}=0.35$.

TABLE I: Vehicle Spec. for Simulation \& Experiments

\begin{tabular}{cccc}
\hline$m$ & {$[\mathrm{~kg}]$} & 2270 & Vehicle mass \\
$I_{z}$ & {$\left[\mathrm{~kg} \cdot \mathrm{m}^{2}\right]$} & 4650 & Vehicle moment of inertia \\
$I_{w}$ & {$\left[\mathrm{~kg} \cdot \mathrm{m}^{2}\right]$} & 1.68 & Wheel moment of inertia \\
$L_{w b}$ & {$[\mathrm{~m}]$} & 2.85 & Vehicle wheel base \\
$b_{f}, b_{r}$ & {$[\mathrm{~m}]$} & $1.42,1.43$ & Front \& rear axles to CG \\
$R_{e}$ & {$[\mathrm{~m}]$} & 0.33 & Effective radius \\
$h_{r c}$ & {$[\mathrm{~m}]$} & 0.54 & Roll axis height \\
$h_{C G}$ & {$[\mathrm{~m}]$} & 0.65 & CG height \\
$T_{f}, T_{r}$ & {$[\mathrm{~m}]$} & $1.62,1.56$ & Front and rear track width \\
\hline
\end{tabular}

For the purpose of analysis, road tests are performed, and results of the longitudinal velocity and force estimators are presented for different road frictions. Measured signals are communicated using a CAN-bus, a conventional communication method in production vehicles. Real-time logging of this sensory information was done using the

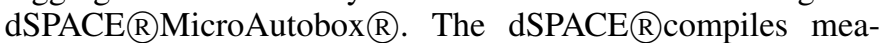
surements for MATLAB/SIMULINK, and the controller provides control signals for the dSPACERas well. The test platform SUV and input/output layout are shown in Fig. 5. To validate the estimated forces, this vehicle is equipped with additional sensors for direct measurement of tire forces and moments at each corner.

Several driving scenarios such as acceleration/deceleration, lane change (LC), double lane change (DLC), acceleration-inturn (AiT), brake-in-turn (BiT), harsh steering, and launches were examined on various roads.

\section{A. Force estimation results}

In Fig. 6-a forces estimated by both the UKF and the unknown input observer (UIO) are compared with the CarSim tire forces on a slippery road for a maneuver with successive accelerator and brake pedal requests. The simulation occurs on a slippery road with $\mu=0.3$, and the initial longitudinal velocity of the vehicle is $u_{0 x}=30 \mathrm{kph}$.

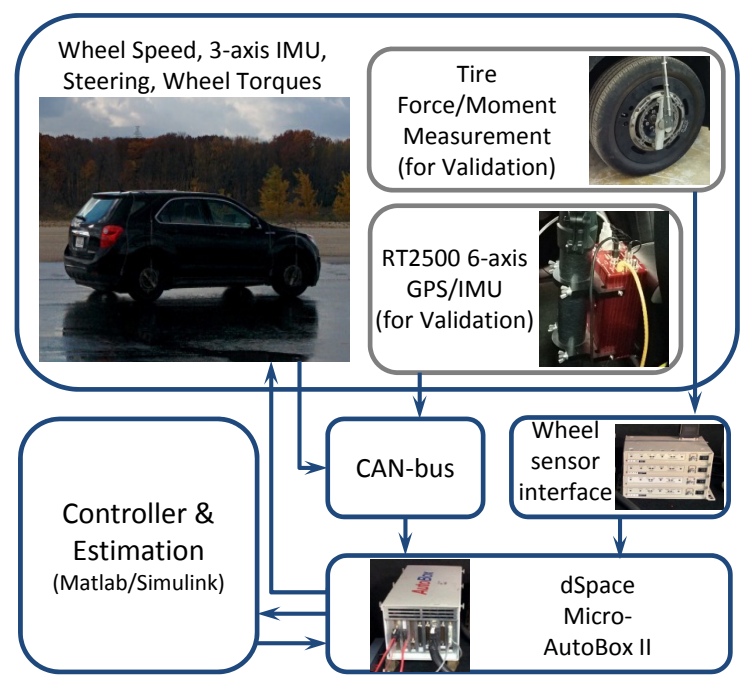

Fig. 5: The fully electrified 4WD test platform and I/O layout
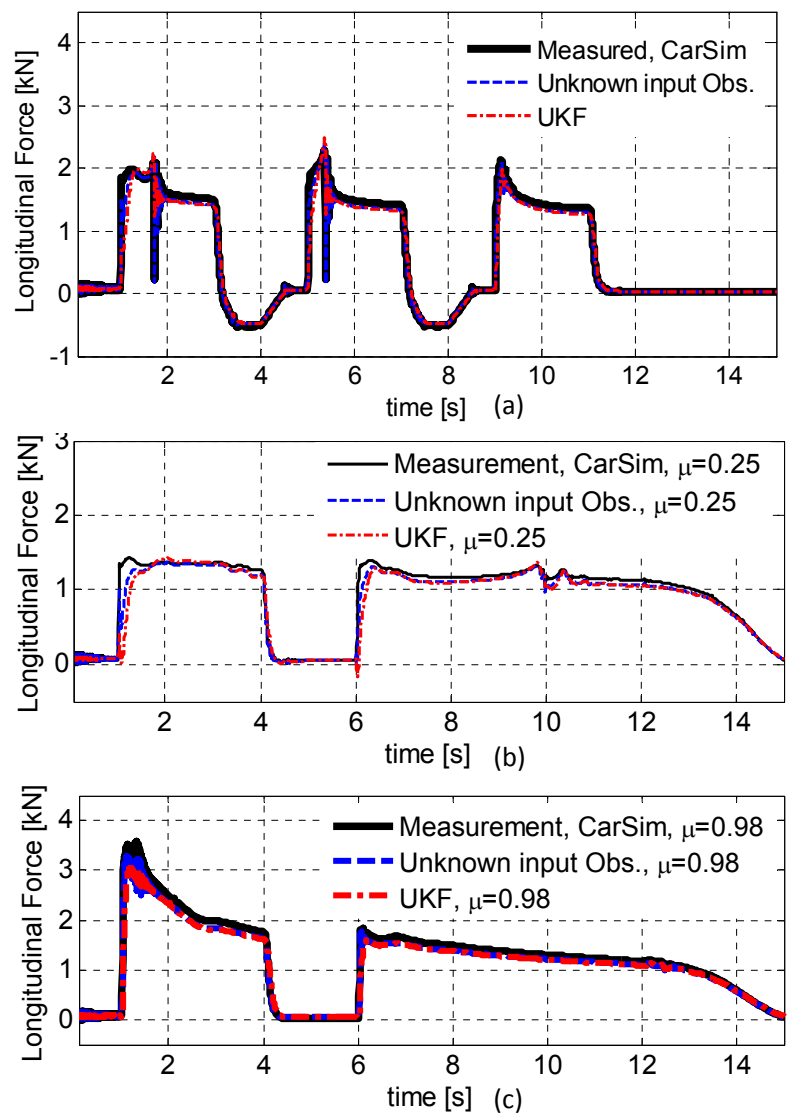

Fig. 6: Estimated longitudinal forces in (a) acceleration/brake on a road with $\mu=0.3$ (b) AiT on dry (c) AiT on a slippery road with $\mu=0.25$ 
The proposed force estimation methods are independent of the road condition and The UKF estimator exhibit more accurate and smooth performance than UIO for such maneuver with successive sign changes of the slip-ratio. An accelerationin-turn (AiT) maneuver on dry and slippery roads is simulated in the CarSim and results are graphically illustrated in Fig. 6-b.

For the AiT driving scenario, the accelerator is applied to $100 \%$ at $t=2[s]$ and continues till $t=4[s]$. It is released between 4 and $6[s]$ and pushed up to $100 \%$ again as a step signal till $t=11[s]$, then it is linearly reduced to zero at $t=15[\mathrm{~s}]$. A steering angle $\delta_{s w}=1[\mathrm{rad}]$ is imposed between $t=2-12[s]$ as well and the steering ratio is $r_{\delta}=16.7$. The performed combined-slip AiT maneuver is harsh, but the suggested UKF for estimator by weighted averaging of the sigma points' values handles the oscillations in the transient regions resulted from imposing and releasing torques on wheels better than UIO. The fluctuations observed in the CarSim's force profile curves are attributed to the requested acceleration with high magnitude.

The road experiment results of the force estimation at the rear-left corner in a driving condition with harsh steering on a wet road $(\mu \approx 0.45)$ are shown in Fig. 7 and compared with the measurement for the test platform vehicle with the stability control system, which prevents high side slip angles.
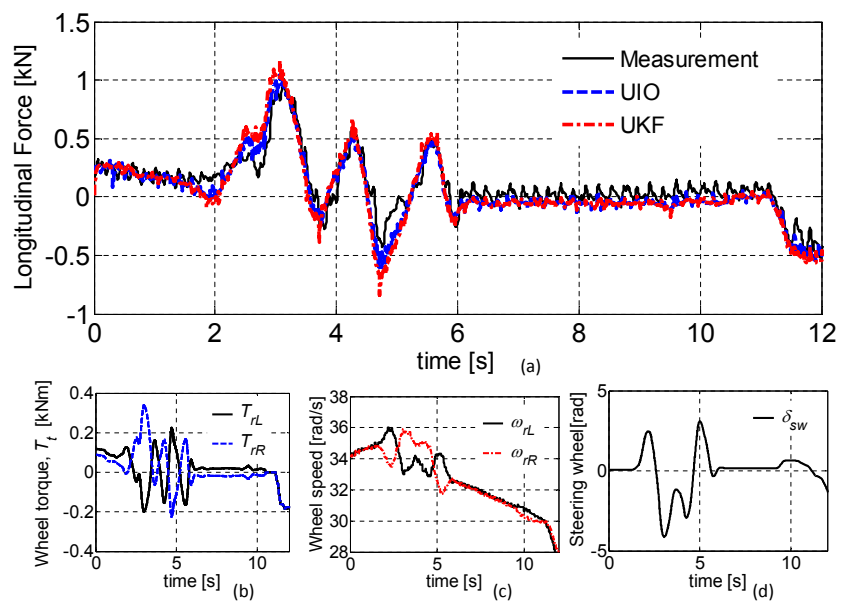

Fig. 7: Road experiments, harsh steering and lane change on wet (a) estimated $\hat{F}_{x}$ at rL (b) rear wheel torques (c) rear wheel speeds (d) steering wheel angle $\delta_{s w}$

The Effective torque and the wheel speed of the rear wheels $T_{t_{r j}}, \omega_{r j}$ are also depicted in Fig. 7. UKF-based and UIO estimators suggest accurate estimation for such harsh steering condition. Another test, a double lane change (DLC) maneuver, with high slip and lateral excitation is conducted on a snowy surface and force estimation results of UIO and the proposed UKF approach are compared in Fig. 8 for the rear left wheel. The force peak values in the DLC maneuver (Fig. 8) on snow is higher than the wet surface (Fig. 7), which shows that the driving on the wet road was not very severe.

There is a certain level of correspondence between the outcomes of the two estimation methods and the measurements,
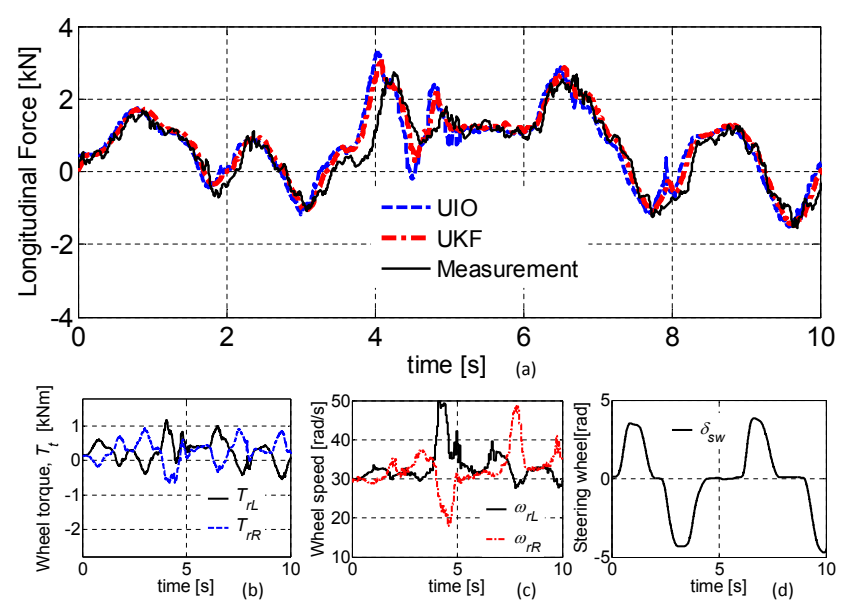

Fig. 8: DLC on snow and experimental results (a) estimated $\hat{F}_{x}$ at $\mathrm{rL}$ (b) rear wheel torques (c) rear wheel speeds (d) steering wheel angle $\delta_{s w}$

even with presence of high slips. However, but UKF exhibits more consistent and smooth results for the DLC on snow from $t=4.1$ to $t=4.5[s]$ and around $t=5.1,7.8[s]$ which are the sudden drop or increase in the torques at each corner.

Accuracy of the longitudinal force estimators are evaluated in different maneuvers with the normalized root mean square of the error NRMS defined by $\bar{\varsigma}=\frac{\sqrt{\sum_{i=1}^{N_{p}}\left(\hat{p}_{i}-p_{i}\right)^{2} / N_{p}}}{p_{m}}$ where the measured and estimated signals are denoted by $p$ and $\hat{p}$ respectively, $N_{p}$ is the number of collected signal samples during a driving scenario (DLC, AiT, BiT, etc.), and $p_{m}=\max _{i=1 \ldots N_{p}}\left|p_{i}\right|$ shows the maximum value of the measured signal. The normalized root mean square $\bar{\varsigma}$ of the estimated longitudinal and vertical forces and velocities in different driving scenarios and on various road frictions are listed in Table II where $\bar{\varsigma}_{1}$ and $\bar{\varsigma}_{2}$ represent the NRMS for the UKF and UIO respectively.

TABLE II: NRMS of the Errors for the Longitudinal Force Estimator (with $\bar{\varsigma}_{1}, \bar{\varsigma}_{2}$ for the UKF and UIO) and Vertical Force Estimator

\begin{tabular}{l|cccccc}
\hline \multirow{2}{*}{$\begin{array}{l}\text { Estimated } \\
\text { forces }\end{array}$} & \multicolumn{2}{|c}{ Steer-wet/dry } & \multicolumn{2}{c}{ DLC-snow } & \multicolumn{2}{c}{ BiT/Accel-snow } \\
\cline { 2 - 7 } & $\bar{\varsigma}_{1}[\%]$ & $\overline{\varsigma_{2}}[\%]$ & $\bar{\varsigma}_{1}[\%]$ & $\bar{\varsigma}_{2}[\%]$ & $\bar{\varsigma}_{1}[\%]$ & $\bar{\varsigma}_{2}[\%]$ \\
\hline$F_{x_{f L}}$ & 3.85 & 4.05 & 4.87 & 6.11 & 5.04 & 4.90 \\
$F_{x_{f R}}$ & 4.02 & 3.91 & 5.32 & 5.18 & 6.23 & 6.28 \\
$F_{x_{r L}}$ & 3.97 & 4.26 & 4.76 & 5.22 & 5.77 & 6.33 \\
$F_{x_{r R}}$ & 4.51 & 4.71 & 4.95 & 5.49 & 5.64 & 7.10 \\
\hline & $\bar{\zeta}[\%]$ & $p_{m}[k N]$ & $\bar{\varsigma}[\%]$ & $p_{m}[k N]$ & $\bar{\varsigma}[\%]$ & $p_{m}[k N]$ \\
\hline$F_{z_{f L}}$ & 3.34 & 12.77 & 3.65 & 9.91 & 3.88 & 9.10 \\
$F_{z_{f R}}$ & 2.72 & 12.41 & 3.01 & 10.16 & 3.03 & 7.06 \\
$F_{z_{r L}}$ & 2.15 & 10.02 & 2.74 & 8.37 & 3.54 & 7.19 \\
$F_{z_{r R}}$ & 1.93 & 10.14 & 2.82 & 8.39 & 2.92 & 6.82 \\
\hline \multicolumn{7}{|c|}{}
\end{tabular}

Table II substantiates that the NRMS of the estimated longitudinal forces by UKF is better than the UIO and it is less than $6.25 \%$ for the performed maneuvers on dry, wet, and snowy roads. Therefore, the UKF approach is selected 
as the longitudinal force estimator for the velocity estimation in Section III because of its superior performance. The moving sigma points (in the UKF approach) through the wheel dynamics (1) reduce estimation fluctuations, especially during transient regions, even in the presence of uncertainties such as in road conditions, which may vary from icy to dry (i.e. $0.1 \leq \theta \leq 0.97)$ in the tire model, effective radii with $\pm 5 \%$ variation, and corrupted measurements of wheel speed and torque with variance $R_{\omega}=0.18$ and $R_{T_{t}}=32$ respectively.

The vertical force estimator leads to the normalized error $\varepsilon \leq 3.9 \%$ which confirms effectiveness of the algorithm on dry and slippery roads. Observed errors between the measured and estimated forces in Table II may have several sources such as camber angle, which has not been modeled in the estimation algorithm. Moreover, inaccurate inertial parameters and uncertainties in the CG location contribute to such errors.

\section{B. Velocity estimation results}

The stochastic observability of the suggested velocity estimator (19) was studied in the previous section for both known and uncertain initial states/covariances, and it is experimentally concluded that for the case with uncertain initial covariance, in addition to the observability condition, two supplementary criteria $\lambda_{\max }\left(\mathcal{M}_{f}\right)=0$ (test1) and $\lambda_{\max }\left(\mathcal{N}_{k+1}\right)<\lambda_{b}$ (test2) are met for various maneuvers and road frictions.

To verify the proposed velocity estimator in maneuvers with lateral excitation, a step steer (SS) scenario is simulated in CarSim with the initial speed $u_{x 0}=60[k p h]$. Fig. 9 illustrates longitudinal velocity estimates for this SS case on a dry road with steering wheel angle $\delta_{s w}=2[\mathrm{rad}]$ at $t=2[\mathrm{~s}]$. The simulation confirms that the newly proposed corner-based velocity estimator provides accurate results in maneuvers with both longitudinal and lateral slips on dry road conditions.

The second set of analysis on the velocity estimator examines the suggested approach in CarSim for an AiT maneuver. Figure 9 also demonstrates an AiT test with the initial velocity of $u_{x 0}=30[\mathrm{kph}]$ and maximum steering wheel angle $\delta_{s w}=1[\mathrm{rad}]$ at $t=2[\mathrm{~s}]$ on dry and slippery $(\mu=0.3)$ roads. Accelerator is applied to $100 \%$ at $t=5[\mathrm{~s}]$ and continues till $t=10[s]$.

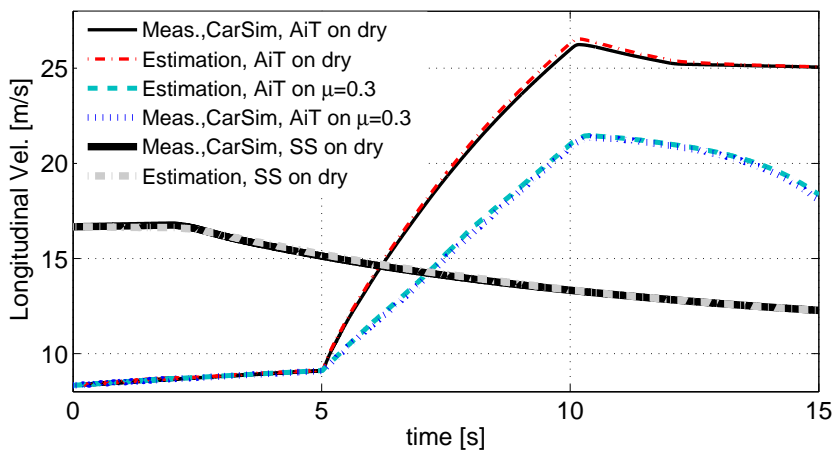

Fig. 9: Estimated longitudinal velocity for SS and AiT on dry and slippery roads
Simulation results reveal that the proposed estimator performs well on various road conditions for maneuvers with both longitudinal and lateral excitations. Outcomes of the road experiments on a fully electrified SUV (shown in Fig. 5) are presented in the followings. One of the main objectives of such an estimator is to provide reliable longitudinal velocity $\hat{u}_{x t}$ at each corner for traction control systems during launch (or hard acceleration) on slippery roads. Fig. 10-a shows performance of the proposed estimator for a launch on a highly slippery wet surface with $\mu \approx 0.3$ which ended on a dry surface with a break. Moreover, an acceleration-in-turn (AiT) maneuver on the same wet surface with a break on dry was performed and estimated longitudinal speed results are provided in Fig. 10-a.
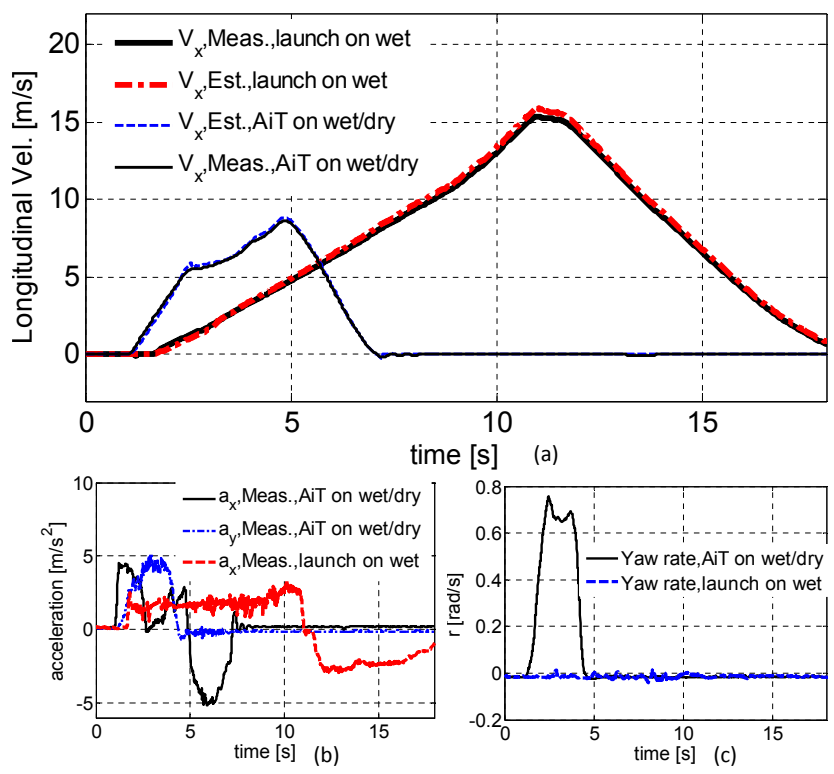

Fig. 10: Launch and AiT on wet and transition to dry (a) estimated speed (b) measured accelerations (c) measured yaw rate

The input torque from the driver for the launch maneuver on highly slippery wet surface brings the tire up to their longitudinal capacity. The measured longitudinal acceleration shown in Fig. 10-b for this maneuver is bellow $3.3\left[\mathrm{~m} / \mathrm{s}^{2}\right]$ which confirms slippery conditions according to teh required accelerator pedal by the driver up to the tires' limits. For both tests, the stability and traction controllers were active, but intentionally set to have a poor performance, which leads to sudden increase in the wheel speed and subsequently slip ratio at each corner. Front tire loose grip in launch and acceleration cases due to the drop in the vertical force on the front track by the load transfer. Therefore, in such maneuvers high slip ratio for the front tires is a concern for traction control systems. Wheel speed for the front tires i.e. $f L, f R$ are shown in Fig. 11 and compared with the estimated velocities and the measured velocities by GPS.

Results of such maneuvers corroborates that even with presence of high slips, the proposed estimator provides accurate 

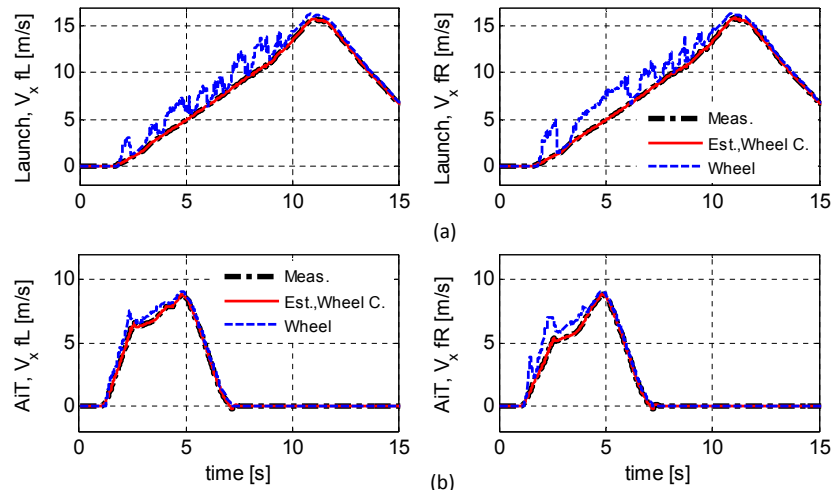

Fig. 11: Wheel speed, measured corner velocities, and estimated velocities at wheel centers (Wheel C.) for (a) launch on wet then dry (b) AiT on wet then dry

and reliable longitudinal velocity estimates $\hat{u}_{x t_{i j}}$ at each tire (Wheel C.) and subsequently at the vehicle CG i.e. $\hat{u}_{x}$.

In order to assess the proposed approach in road experiments with high lateral excitation (combined-slip characteristics) and large longitudinal slip, a brake-in-turn (BiT) accompanied by hard acceleration on snow (with $\mu \approx 0.35$ ) is done and the estimation results are provided in Fig. 12. Measured accelerations and the yaw rate for such BiT case is provided in Fig. 12-b that shows the weak grip condition for each tire.
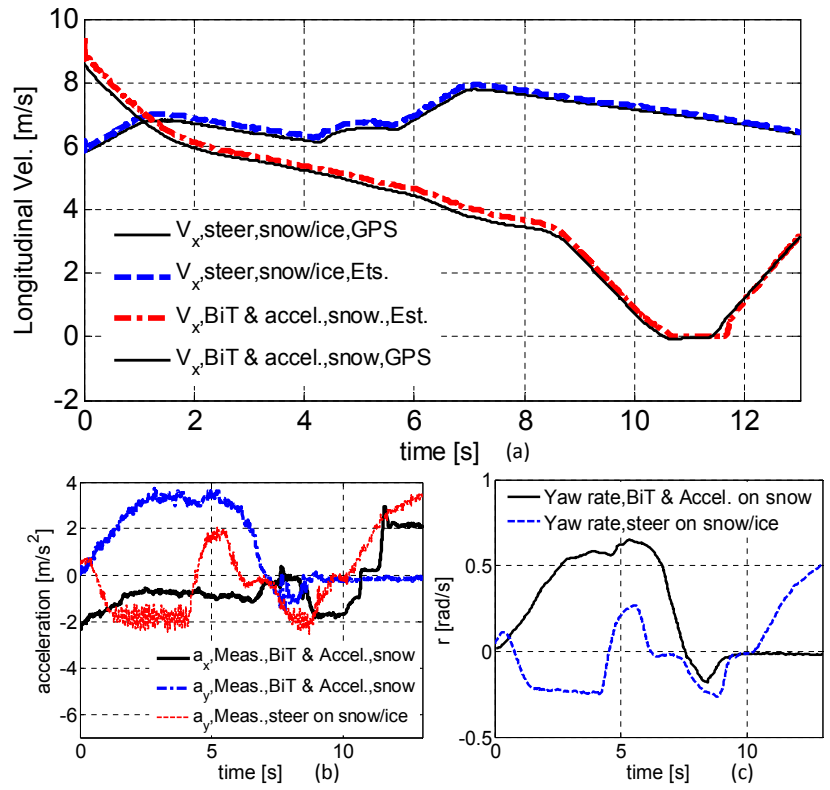

Fig. 12: $\hat{u}_{x}, \mathrm{BiT}$ and steering on snow/ice

Another experiment with harsh steering on snow and ice road (with $\mu \approx 0.25$ ) was executed to validate the method and the outcomes are also demonstrated in Fig. 12 together with the measured accelerations and the yaw rate. As can be seen from Fig. 12-a, the developed algorithm with the high- slip detection module provides accurate velocity estimates in maneuvers with combined-slip characteristics on snowy and icy roads as well.

Wheel speed of the front tires' center for the BiT and steering maneuvers on snow/ice are compared with the estimated and measured velocities in Fig. 13. The estimates by the proposed corner-based approach have correspondence with the measurement in spite of the large slip cases around $t=6[s]$ and after $t=11[\mathrm{~s}]$ for the harsh steer on snow/ice and in $4 \leq t \leq 7.8[s]$ for BiT on snow.
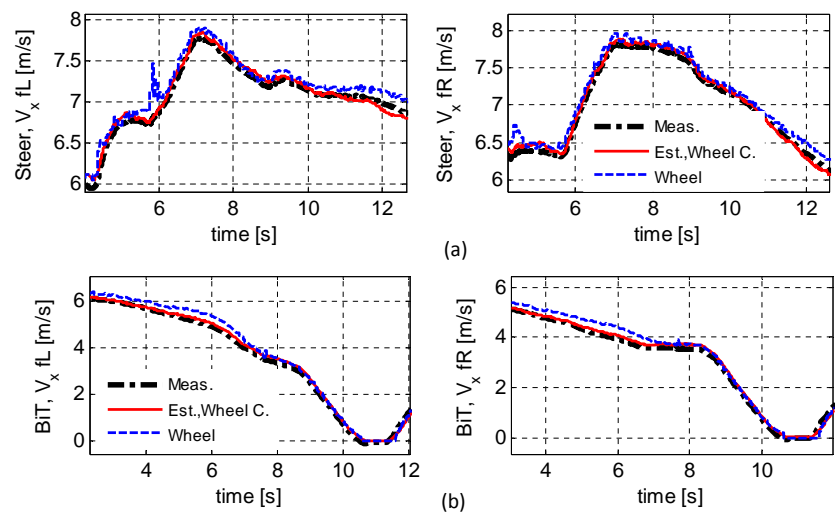

(a)

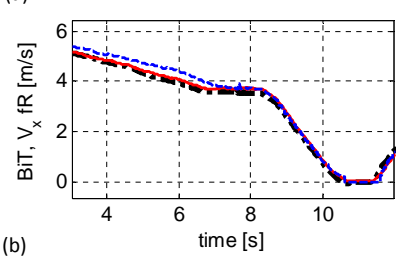

Fig. 13: Wheel speed, measured corner velocities, and estimated velocities at Wheel C.for (a) steering on snow/ice (b) BiT and Accel. on snow

The high-slip detection module changes the covariance matrices of the Kalman-based estimator based on the methodology discussed in section III. This significantly improves the outcomes by defining the level of reliance on the forces for the velocity estimation correction.

The simulation and experimental results provided in this section show that the suggested method with the Kalman observer can provide longitudinal state estimates, in the absence of road friction details. This method has been experimentally tested with aggressive maneuvers such as increasing the longitudinal speed during cornering, acceleration-in-turn, harsh steering, and launch on low-friction surfaces.

\section{CONClusion}

A corner-based longitudinal state estimation robust to the road condition changes is proposed in this article. An unknown input observer and unscented Kalman filter are investigated for estimation of longitudinal forces and the stability of the unknown input observer for longitudinal force estimation is provided. The important feature of the developed corner-based force estimator is that it does not implement any tire model and is independent from changes in the road friction or tire parameters due to wear, inflation pressure, etc. In addition, the suggested force estimators can address the cases in which tires are on surfaces with various road frictions (split- $\mu$ ).

A Kalman-based velocity estimation method using the average Lumped LuGre model at each corner is proposed in this article, and its performance is studied. Taking advantage of 
the dynamics on the internal tire deflection states, the LuGre model facilitates combination of the vehicle kinematics and tire forces. Stochastic observability of the proposed Kalman-based velocity estimator are also investigated. Based on the experimental and simulation results, it is concluded that the proposed estimator can handle dry/slippery roads with pure/combinedslip conditions.

One of significant advantages of the suggested Kalman observer is that the unidirectional lumped LuGre model can be employed instead of the combined-slip model. This substitution is made possible by denoting the term $z \frac{\sigma_{0}\left|V_{r}\right|}{\theta g\left(V_{r}\right)}$, which contains the combined friction model, as uncertainty.

The proposed velocity estimation algorithm detects large slip ratio cases with an adaptive high-slip threshold (29), based on the excitation level, to allocate adaptive covariance matrices and tackle the noises associated with harsh maneuvers. The road experiments show that the corner-based state estimators can handle dry and slippery roads with the normalized error $R M S \bar{\varsigma}<6.25 \%$ for the longitudinal force estimation, $\bar{\varsigma}<$ $3.9 \%$ for the vertical (normal) force, and $\bar{\varsigma}<6.6 \%$ for the longitudinal velocity estimation.

The developed longitudinal estimators can be integrated with active safety systems (e.g. stability control and traction control) to enhance the performance of such systems in presence of road friction changes. Moreover, while preserving the overall structure of the estimation, one can replace force and velocity estimators independently because of the modularity of the provided structure.

Observed inconsistencies in some tests of the proposed estimation approach are due to the lack of a general chassis model, a limitation to be addressed in future works, using the vehicle generalized force model.

\section{ACKNOWLEDGEMENT}

This work was supported by Automotive Partnership Canada, Ontario Research Fund, and the General Motors Co. [grant numbers APCPJ 39599609 and ORF-RE-04-039].

\section{APPENDIX}

- Detectability and stabilizability:

Definition 1: The pair $\left[A_{k}, C_{k}\right]$ in the linear timevarying discrete-time system with state update $x_{k+1}=$ $A_{k} x_{k}+B_{k} u_{k}$ and output equation $y_{k}=C_{k} x_{k}$ is uniformly detectable if $\exists 0 \leq c_{1} \leq 1, c_{2} \in \mathbb{R}^{+}$and $q, k_{2} \geq 0$, such that in case $\left\|\phi_{k_{1}+q, k_{1}} \vartheta\right\| \geq c_{1}\|\vartheta\|$ for some $\vartheta, k_{1}$, then $\vartheta^{T} \mathbb{V}\left(k_{1}, k_{2}\right) \vartheta \geq c_{2} \vartheta^{T} \vartheta$, which necessitates the observability grammian $\mathbb{V}\left(k_{1}, k_{2}\right)$ to be $\mathbb{V}\left(k_{1}, k_{2}\right) \geq d_{1} I>0$ for some $d_{1}[39]$ :

$$
\mathbb{V}\left(k_{1}, k_{2}\right)=\sum_{k=k_{1}}^{k_{2}} \phi_{k, k_{1}}^{T} C_{k}^{T} C_{k} \phi_{k, k_{1}},
$$

where $\phi_{i, j}=\phi_{i, i-1} \phi_{i-1, j}$ and $\phi_{i+1, i}=A_{i}$ as the state transition matrices for $i \geq j$. In addition, the pair $\left[A_{k}, B_{k}\right]$ in the linear time-varying discrete-time system (20) without process and measurement noise effect is stabilizable if $\exists 0 \leq c_{1} \leq 1, c_{2} \in \mathbb{R}^{+}$and $q, k_{2} \geq 0$, such that in case $\left\|\phi_{k 2, k 2-q} \vartheta\right\| \geq c_{1}\|\vartheta\|$ for some $\vartheta$, then $\vartheta^{T} \mathbb{W}\left(k_{1}, k_{2}-1\right) \vartheta \geq c_{2} \vartheta^{T} \vartheta$, which necessitates the controllability grammian $\mathbb{W}\left(k_{1}, k_{2}-1\right)$ to be full rank [44]:

$$
\mathbb{W}\left(k_{1}, k_{2}-1\right)=\sum_{k=k_{1}}^{k_{2}-1} \phi_{k_{2}, k+1} B_{k} B_{k}^{T} \phi_{k_{2}, k+1}^{T},
$$

- Bounded error covariance for the Kalman filter: this characteristic for the time-invariant KF has been proved before, but provided here for convenience. Detectability condition on $(A, C)$ leads to a linear estimator with matrix $K^{*}$ :

$$
x_{k+1 \mid k}^{*}=A x_{k \mid k-1}^{*}+K^{*}\left(y_{k}-C x_{k \mid k-1}^{*}\right),
$$

where $\left(A-K^{*} C\right)$ is stable. Thus, the error covariance matrix for such estimator is defined by $\bar{P}_{k+1 \mid k}^{*} \triangleq$ $\mathbb{E}\left[\left(x_{k+1}-\hat{x}_{k+1 \mid k}^{*}\right)\left(x_{k+1}-\hat{x}_{k+1 \mid k}^{*}\right)^{T}\right]$ that yields::

$$
\bar{P}_{k+1 \mid k}^{*}=\left(A-K^{*} C\right) \bar{P}_{k}^{*}\left(A-K^{*} C\right)^{T}+K^{*} R K^{* T},
$$

which can be written as:

$$
\begin{aligned}
\bar{P}_{k+1 \mid k}^{*} & =\left(A-K^{*} C\right)^{k+1} \bar{P}_{0 \mid-1}^{*}\left(\left(A-K^{*} C\right)^{k+1}\right)^{T} \\
& +\sum_{i=0}^{k}\left(A-K^{*} C\right)^{i}\left(K^{*} R K^{* T}+Q\right)\left(\left(A-K^{*} C\right)^{i}\right)^{T}
\end{aligned}
$$

The first term vanishes and the second term is also bounded because of the stability of $\left(A-K^{*} C\right)$. Therefore, the error covariance $\bar{P}_{k+1 \mid k}^{*}$ of such linear estimator is bounded. This results in bounded error covariance $\bar{P}_{k}$ for the Kalman estimator because of the optimality of the KF.

- Defining $\mathcal{M}_{k+1}$ and $\mathcal{N}_{k+1}$ for completely uncertain initial covariance/states [42]: the initial $\mathcal{M}_{1}, \mathcal{N}_{1}$ are attainable by the initial measurement error covariance $R_{0}$ as $\Xi_{0}^{T} \Xi_{0}=\bar{R}_{0}$, which yields the projector $\Phi$ of a vector onto the orthogonal complement of the range space $\Sigma$ :

$$
\begin{aligned}
\Sigma_{0} & =C_{0}^{T} \Xi_{0}^{-1} \\
\Phi_{0} & =I-\Sigma_{0}\left(\Sigma_{0}^{T} \Sigma_{0}\right)^{*} \Sigma_{0}^{T} \\
X_{0} X_{0}^{T} & =\Phi_{0} \\
\mathcal{M}_{1} & =A_{0} X_{0} X_{0}^{T} A_{0}^{T} \\
\mathcal{N}_{1} & =\bar{Q}_{0}+A_{0} \Sigma_{0}\left(\left(\Sigma_{0}^{T} \Sigma_{0}\right)^{*}\right)^{2} \Sigma_{0}^{T} A_{0}^{T},
\end{aligned}
$$

where $(.)^{*}$ represents pseudo inverse of the matrix (.) and full rank factorization of $\Phi_{0}$ is denoted by $X_{0}$. The matrix $\mathcal{M}_{k+1}$ is then defined using the fresh $C_{k}$ and the 
measurement noise $R_{k}$ as the following procedure:

$$
\begin{aligned}
\Xi_{k}^{T} \Xi_{k} & =\bar{R}_{k}+C_{k} \mathcal{N} C_{k}^{T} \\
\Sigma_{k} & =X_{0, k-1}^{T} \phi_{k, 0}^{T} C_{k}^{T} \Xi_{k}^{-1} \\
\Phi_{k} & =I-\Sigma_{k}\left(\Sigma_{k}^{T} \Sigma_{k}\right)^{*} \Sigma_{K}^{T} \\
X_{k} X_{k}^{T} & =\Phi_{k} \\
\mathcal{M}_{k+1} & =\phi_{k, 0} X_{0, k} X_{0, k}^{T} \phi_{k, 0}^{T}
\end{aligned}
$$

in which $X_{k}$ is the full rank factorization of $\Phi_{k}$ and $X_{0, k} \triangleq X_{0} X_{1} \ldots X_{k}$. Employing the condition $\lambda_{\max }\left(\mathcal{M}_{f}\right)=0$ for a finite time, $\mathcal{N}_{k+1}$ is related to $\mathcal{N}_{k}$ as in (27). In addition, the $\mathcal{S}_{k+1}$ matrix in the simplified Riccati equation $\bar{P}_{k+1 \mid k}=\mathcal{N}_{k+1}+\mathcal{S}_{k+1}$ can be written as:

$$
\begin{aligned}
\mathcal{S}_{k+1} & =A_{k} \mathcal{S}_{k} A_{k}^{T}-A_{k} \mathcal{N}_{k} C_{k}^{T} \Xi_{k}^{-1}\left(\frac{\mathcal{T}_{k, 1}}{\psi}+\ldots\right) \Xi_{k}^{-T} C_{k}{ }^{-} \mathcal{T}_{k, 1} \\
& -A_{k} \mathcal{N}_{k} C_{k}^{T} \Xi_{K}^{-1}(I-\ldots) \Xi_{k}^{-T} C_{k} \mathcal{S}_{k} A_{k}^{T} \\
& -A_{k} \mathcal{S}_{k} C_{k}^{T} \Xi_{K}^{-1}\left(I-\frac{\mathcal{T}_{k, 1}}{\psi}+\ldots\right) \Xi_{k}^{-T} C_{k} \mathcal{N}_{k} A_{k}^{T} \\
& -A_{k} \mathcal{S}_{k} C_{k}^{T} \Xi_{K}^{-1}\left(I-\frac{\mathcal{T}_{k, 1}}{\psi}+\ldots\right) \Xi_{k}^{-T} C_{k} \mathcal{S}_{k} A_{k}^{T}
\end{aligned}
$$

\section{REFERENCES}

[1] J. Ryu and J. C. Gerdes, "Integrating inertial sensors with global positioning system (gps) for vehicle dynamics control," Journal of Dynamic Systems, Measurement, and Control, vol. 126, no. 2, pp. 243254, 2004.

[2] D. M. Bevly, J. Ryu, and J. C. Gerde, "Integrating ins sensors with gps measurements for continuous estimation of vehicle sideslip, roll, and tire cornering stiffness," Intelligent Transportation Systems, IEEE Transactions on, vol. 7, no. 4, pp. 483-493, 2006.

[3] L. Imsland, T. A. Johansen, T. I. Fossen, H. F. Grip, J. C. Kalkkuhl, and A. Suissa, "Vehicle velocity estimation using nonlinear observers," Automatica, vol. 42, no. 12, pp. 2091-2103, 2006.

[4] L. Imsland, H. F. Grip, T. A. Johansen, T. I. Fossen, J. C. Kalkkuhl, and A. Suissa, "Nonlinear observer for vehicle velocity with friction and road bank angle adaptation-validation and comparison with an extended kalman filter," SAE Technical Paper, Tech. Rep., 2007.

[5] Y.-H. J. Hsu, "Estimation and control of lateral tire forces using steering torque," Ph.D. dissertation, Stanford University, 2009.

[6] G. Baffet, A. Charara, and G. Dherbomez, "An observer of tire-road forces and friction for active security vehicle systems," Mechatronics, IEEE/ASME Transactions on, vol. 12, no. 6, pp. 651-661, 2007.

[7] Y.-H. J. Hsu and J. C. Gerdes, "The predictive nature of pneumatic trail: Tire slip angle and peak force estimation using steering torque," in AVECO8, Kobe, Japan, 2008.

[8] J.-H. Yoon and H. Peng, "A cost-effective sideslip estimation method using velocity measurements from two gps receivers," Vehicular Technology, IEEE Transactions on, vol. 63, no. 6, pp. 2589-2599, 2014.

[9] A. Hac and M. D. Simpson, "Estimation of vehicle side slip angle and yaw rate," SAE Technical Paper, Tech. Rep., 2000.

[10] T. A. Wenzel, K. Burnham, M. Blundell, and R. Williams, "Dual extended kalman filter for vehicle state and parameter estimation," Vehicle System Dynamics, vol. 44, no. 2, pp. 153-171, 2006.

[11] S. J. Julier, J. K. Uhlmann, and H. F. Durrant-Whyte, "A new approach for filtering nonlinear systems," in American Control Conference, Proceedings of the 1995, vol. 3. Seattle, WA: IEEE, 1995, pp. 1628-1632.
[12] E. A. Wan and R. Van Der Merwe, "The unscented kalman filter for nonlinear estimation," in Adaptive Systems for Signal Processing, Communications, and Control Symposium 2000. AS-SPCC. The IEEE 2000. Lake Louise, Alberta: IEEE, 2000, pp. 153-158.

[13] W. R. Pasterkamp and H. Pacejka, "The tyre as a sensor to estimate friction," Vehicle System Dynamics, vol. 27, no. 5-6, pp. 409-422, 1997.

[14] R. Uil, "Tyre Models for Steady-State Vehicle Handling Analysis," Ph.D. dissertation, Eindhoven University of Technology, 2007.

[15] S. Antonov, A. Fehn, and A. Kugi, "Unscented kalman filter for vehicle state estimation," Vehicle System Dynamics, vol. 49, no. 9, pp. 14971520, 2011.

[16] G. Magallan, C. H. De Angelo, G. O. Garcia et al., "Maximization of the traction forces in a 2 wd electric vehicle," Vehicular Technology, IEEE Transactions on, vol. 60, no. 2, pp. 369-380, 2011.

[17] C. Canudas-De-Wit and P. Tsiotras, "Dynamic Tire Friction Models for Vehicle Traction Control," in proceedings of the 38th Conference on Decision \& Control, Phoenix, AZ, 1999, pp. 3746-3751.

X. Zhang, Y. Xu, M. Pan, and F. Ren, "A vehicle abs adaptive slidingmode control algorithm based on the vehicle velocity estimation and tyre/road friction coefficient estimations," Vehicle System Dynamics, vol. 52, no. 4, pp. 475-503, 2014.

[19] M. Burckhardt, "Fahrwerktechnik: Radschlupf-regelsysteme," VogelVerlag, Germany, p. 16, 1993.

[20] M. Gadola, D. Chindamo, M. Romano, and F. Padula, "Development and validation of a kalman filter-based model for vehicle slip angle estimation," Vehicle System Dynamics, vol. 52, no. 1, pp. 68-84, 2014.

[21] C. Canudas-de Wit, M. L. Petersen, and A. Shiriaev, "A new nonlinear observer for tire/road distributed contact friction," in Decision and Control, Proceedings. 42nd IEEE Conference on, vol. 3. Maui, HI: IEEE, 2003, pp. 2246-2251.

[22] N. Patel, C. Edwards, and S. Spurgeon, "Tyreroad friction estimationa comparative study," Proceedings of the Institution of Mechanical Engineers, Part D: Journal of Automobile Engineering, vol. 222, no. 12, pp. 2337-2351, 2008.

[23] G. Baffet, A. Charara, and D. Lechner, "Estimation of vehicle sideslip, tire force and wheel cornering stiffness," Control Engineering Practice, vol. 17, no. 11, pp. 1255-1264, 2009.

[24] E. Hashemi, M. Pirani, A. Khajepour, B. Fidan, A. Kasaiezadeh, S. Chen, and B. Litkouhi, "Integrated estimation structure for the tire friction forces in ground vehicles," in Advanced Intelligent Mechatronics, 2016 IEEE Conference on, Banff, Canada, 2016.

[25] M. Doumiati, A. Victorino, D. Lechner, G. Baffet, and A. Charara, "Observers for vehicle tyre/road forces estimation: experimental validation," Vehicle System Dynamics, vol. 48, no. 11, pp. 1345-1378, 2010.

[26] E. Hashemi, A. Kasaeizadeh, A. Khajepour, N. Mushchuk, and S.-K. Chen, "Robust Estimation and Experimental Evaluation of Longitudinal Friction Forces in Ground Vehicles," in ASME IMECE2014, Montreal, Canada, 2014.

[27] S. Mammar, S. Glaser, and M. Netto, "Vehicle lateral dynamics estimation using unknown input proportional-integral observers," in American Control Conference, 2006. Minneapolis, MN: IEEE, 2006, pp. 6-pp.

[28] Y. Wang, D. M. Bevly, and S.-k. Chen, "Longitudinal tire force estimation with unknown input observer," in ASME 2012 5th Annual Dynamic Systems and Control Conference. Fort Lauderdale, FL: American Society of Mechanical Engineers, 2012, pp. 523-530.

[29] S. Hong, T. Smith, F. Borrelli, and J. K. Hedrick, "Vehicle inertial parameter identification using extended and unscented kalman filters," in Intelligent Transportation Systems-(ITSC), 2013 16th International IEEE Conference on. Hague, Netherlands: IEEE, 2013, pp. 14361441 .

[30] S. Hong, C. Lee, F. Borrelli, and J. K. Hedrick, "A novel approach for vehicle inertial parameter identification using a dual kalman filter," Intelligent Transportation Systems, IEEE Transactions on, vol. 16, no. 1, pp. 151-161, 2015. 
[31] E. Haykin, Simon, Kalman Filtering and Neural Networks. New York: John Wiley \& Sons, Inc., 2004, vol. 47.

[32] A. Rehm, "Estimation of vehicle roll angle," in Communications, Control and Signal Processing (ISCCSP), 2010 4th International Symposium on. Limassol, Cyprus: IEEE, 2010, pp. 1-4.

[33] H. Pacejka and I. Besselink, "Magic formula tyre model with transient properties," Vehicle system dynamics, vol. 27, no. S1, pp. 234-249, 1997.

[34] C. Canudas-de Wit, P. Tsiotras, E. Velenis, M. Basset, and G. Gissinger, "Dynamic friction models for road/tire longitudinal interaction," Vehicle System Dynamics, vol. 39, no. 3, pp. 189-226, 2003.

[35] Y. Wang and D. Bevly, "Longitudinal vehicle state estimation," General Motors Summary Report, Tech. Rep., 2010.

[36] A. E. Bryson and Y.-C. Ho, Applied optimal control: optimization, estimation and control. Levittown, PA: Taylor Francis, 1975.

[37] P. R. Kumar and P. Varaiya, Stochastic systems: estimation, identification and adaptive control. New York: Prentice-Hall, Inc., 1986.

[38] G. F. Franklin, J. D. Powell, and M. L. Workman, Digital Control of Dynamic Systems. Menlo Park: Addison Wesley Longman, 1998.

[39] B. Anderson and J. B. Moore, "Detectability and stabilizability of timevarying discrete-time linear systems," SIAM Journal on Control and Optimization, vol. 19, no. 1, pp. 20-32, 1981.

[40] R. F. Stengel, Optimal control and estimation. New York: Courier Corporation, 2012.

[41] B. Delyon, "A note on uniform observability," Automatic Control, IEEE Transactions on, vol. 46, no. 8, pp. 1326-1327, 2001.

[42] V. L. Bageshwar, D. Gebre-Egziabher, W. L. Garrard, and T. T. Georgiou, "Stochastic observability test for discrete-time kalman filters," Journal of Guidance, Control, and Dynamics, vol. 32, no. 4, pp. 13561370, 2009.

[43] R. Tóth, Modeling and identification of linear parameter-varying systems. Berlin Heidelberg: Springer, 2010, vol. 403.

[44] B. D. Anderson and J. B. Moore, Optimal filtering. New York: Dover Publications, 1995 